\title{
A Comparison between Mouse, In Silico, and Robot Odor Plume Navigation Reveals Advantages of Mouse Odor Tracking
}

\author{
A. Gumaste, ${ }^{1,2,3}$ G. Coronas-Samano, ${ }^{2,3}$ J. Hengenius, ${ }^{4}$ R. Axman, ${ }^{2}$ ㅌ․ G. Connor, ${ }^{5}$ ㄴ. L. Baker, ${ }^{2,3}$ B. \\ Ermentrout, $^{4}$ (1) J. P. Crimaldi, ${ }^{5}$ and ${ }^{10}$ J. V. Verhagen ${ }^{1,2,3}$
}

https://doi.org/10.1523/ENEURO.0212-19.2019

${ }^{1}$ Interdepartmental Neuroscience Program, Yale University, New Haven, CT $06510,{ }^{2}$ The John B. Pierce Laboratory, New Haven, CT 06519, ${ }^{3}$ Department of Neuroscience, Yale School of Medicine, New Haven, CT 06510, ${ }^{4}$ Department of Mathematics, University of Pittsburgh, Pittsburgh, PA 15260, and ${ }^{5}$ Department of Civil, Environmental and

Architectural Engineering, University of Colorado, Boulder, CO 80309

\begin{abstract}
Localization of odors is essential to animal survival, and thus animals are adept at odor navigation. In natural conditions animals encounter odor sources in which odor is carried by air flow varying in complexity. We sought to identify potential minimalist strategies that can effectively be used for odor-based navigation and asses their performance in an increasingly chaotic environment. To do so, we compared mouse, in silico model, and Arduino-based robot odor-localization behavior in a standardized odor landscape. Mouse performance remains robust in the presence of increased complexity, showing a shift in strategy towards faster movement with increased environmental complexity. Implementing simple binaral and temporal models of tropotaxis and klinotaxis, an in silico model and Arduino robot, in the same environment as the mice, are equally successful in locating the odor source within a plume of low complexity. However, performance of these algorithms significantly drops when the chaotic nature of the plume is increased. Additionally, both algorithm-driven systems show more successful performance when using a strictly binaral model at a larger sensor separation distance and more successful performance when using a temporal and binaral model when using a smaller sensor separation distance. This suggests that with an increasingly chaotic odor environment, mice rely on complex strategies that allow for robust odor localization that cannot be resolved by minimal algorithms that display robust performance at low levels of complexity. Thus, highlighting that an animal's ability to modulate behavior with environmental complexity is beneficial for odor localization.
\end{abstract}

Key words: in silico; mouse; navigation; odor plume; robot; turbulence

\section{Significance Statement}

A promising body of work has been devoted to designing robots and algorithms that address the strategies used by animals during odor-based navigation. One method to do so is by designing models that account for complex navigational tactics implemented by a particular species. How do these models directly compare to animal behavior in the same environment? We addressed this question by comparing odorlocalization performance of minimal spatial and temporal algorithms in silico and in a robot to the strategies and performance of mice in the same odor environment. Through implementing this unique comparison, we revealed that mouse behavior remains robust with an increase in odor plume complexity, whereas simple algorithm behavior (although high performing at low plume complexity) does not. 


\section{Introduction}

Odor-based navigation is critical to animal survival as animals depend on olfactory cues to locate food sources, find mates, and avoid predators. Odors in nature are often carried by chaotic air or water flow, producing plumes with complex spatiotemporal structure. In large naturalistic environments, odor plumes become characterized by odor fluctuations, providing animals with a dynamic odor environment to navigate (Crimaldi et al., 2002; Connor et al., 2018).

Animals display a variety of behavioral strategies when navigating odor landscapes. Mammals exhibit zigzagging casting behavior when tracking odor trails (Porter et al., 2007; Khan et al., 2012; Jones and Urban, 2018; Liu et al., 2019) and similarly, insects display casting behavior when traveling through airborne odor plumes (Willis and Avondet, 2005; Gomez-Marin et al., 2011). For both insects and crustaceans, odor plume complexity can affect odor-source localization (Mafra-Neto and Cardé, 1994; Keller and Weissburg, 2004). Moths exhibit a decrease in casting behavior and increase in fast, straight upwind paths in the presence of increased complexity, suggesting that complexity can be beneficial for odor tracking in some species. Although insect and crustacean behavior within odor landscapes has been studied for decades, a small but growing body of literature is focusing on the behavioral strategies used by mammals, specifically rodents, for airborne odor source localization. When rodents are tested on odor source localization in small flowchambers where odor is released from a set of predictable locations, they ultimately predominantly use a habitual strategy relying on spatial memory to find odor ports (Bhattacharyya and Bhalla, 2015; Gire et al., 2016). Additionally, these studies suggest that rodents do not exhibit casting behavior during odor localization within airborne plumes, an interesting contrast to the casting observed during trail following.

To systematically determine the strategies that may account for animal odor-based navigation, scientists have turned to robotics. Several robotics-based approaches to odor localization have focused on replicating well-studied

Received June 4, 2019; accepted December 19, 2019; First published January 10, 2020.

The authors declare no competing financial interests.

Author contributions: A.G., G.C.-S., J.H., R.A., K.L.B., and J.V.V. designed research; A.G., G.C.-S., J.H., E.G.C., J.P.C., and J.V.V. performed research; A.G., G.C.-S., J.H., B.E., and J.V.V. analyzed data; A.G., J.H., and J.V.V. wrote the paper.

This work was supported by National Institutes of Health/National Institute on Deafness and Other Communication Disorders Grants R01 DC011286, R01 DC014723, and NSF BRAIN 1555880 (to J.V.V.), NSF BRAIN 1555916 (to B.E.), and NSF BRAIN 1555862 (to J.P.C.)

Acknowledgements: We thank the technical support of J. Buckley, R. Goodman, A. Wilkins, and T. D'Alessandro of the John B. Pierce Shop for creating the flow chamber.

Correspondence should be addressed to J. V. Verhagen at jverhagen@jbpierce.org.

https://doi.org/10.1523/ENEURO.0212-19.2019

Copyright (C) 2020 Gumaste et al.

This is an open-access article distributed under the terms of the Creative Commons Attribution 4.0 International license, which permits unrestricted use, distribution and reproduction in any medium provided that the original work is properly attributed. moth navigational strategies. These studies employed algorithms combining odor and wind-sensing to mimic casting behavior (Ishida et al., 1996; Harvey et al., 2008; Lochmatter et al., 2008; Lochmatter and Martinoli, 2009). Successful robotics strategies have implemented fans to actively draw air into sensors, similar to the beating of a moth's wings, showing that fanning action causes a greater difference in perceived concentration between two sensors (Nakamoto et al., 1996). Although implementing robotic algorithms inspired by animal trajectories is useful when developing robust odor-source localization strategies, it is critical that the efficacy of these algorithms is tested through direct comparison with animals. Studies aimed at bridging the gap between simulations and real animal behavior have used insect antennas to replace sensors as well as used a robot to generate lobster antenna movements to study the resulting changes to the odor environment (Kuwana and Shimoyama, 1998; Koehl et al., 2001). Stereo smell is beneficial for odor localization in invertebrates and mammals alike (Porter et al., 2007; Catania, 2013; Jones and Urban, 2018). With unilateral naris occlusion, mouse odor localization accuracy drops and when input to one antenna is blocked, Drosophila fail to orient toward airborne odor plumes (Rajan et al., 2006; Duistermars et al., 2009). Thus, when developing algorithms to compare to animal odor-navigation behavior, it is essential to consider stereo smell. When tested in identical physical conditions to the milieu of a lobster, a RoboLobster implementing minimal algorithms based on a difference in concentration between two chemical sensors, displays paths that are both more tortuous and less successful when compared to an actual lobster (Grasso et al., 2000). This suggests that lobster odor-navigation strategy is more complex than a simple comparison between concentrations at two sensors.

Here, we directly compare the behavior of mice, minimal in silico odor-localization models, and an Arduino robot implementing these models (tropotaxis and klinotaxis) in the presence of two levels of odor plume complexity. The use of in silico models allows for flexibility of testing a variety of navigation strategies, supports the quantification of effects of varied sensor parameters and enables the measurement of instantaneous concentration during odor navigation. To the best of our knowledge, our study is the first to directly test airborne odor-navigation algorithms, designed in silico, implemented in a robot and real rodent behavior within the same flow chamber. We find that mouse odor localization remains robust in a plume which is increasingly chaotic, and that complexity may benefit the efficiency of navigation. Additionally, we find that when tested in the same environment as the mouse, an Arduino robot shows decreased performance with increased odor plume complexity, highlighting the robustness of mouse navigation behavior.

\section{Materials and Methods}

\section{Standard odor landscape (SOL)}

A SOL arena was built as described in Connor et al. (2018), barring a few adjustments related to the behavioral assay. The core of the flow chamber had dimensions of 
$100 \mathrm{~cm}$ wide, $100 \mathrm{~cm}$ long (in flow direction), and $30 \mathrm{~cm}$ tall. The chamber was flanked by honeycomb flowstraighteners (Plascore PC2-125-W-2 polycarbonate 1/8inch cell, 2 inches thick, $1 \times 0.3 \mathrm{~m}$ ) and the air inlet had a turbulence grid $(2.5 \times 2.5 \mathrm{~cm}$ spacing, steel grid wire $3-\mathrm{mm} \mathrm{OD)} 20 \mathrm{~cm}$ downstream of the inlet honeycomb (Fig. 1A). Airflow of $5 \mathrm{~cm} / \mathrm{s}$ was established using a vacuum attached to the outlet of the flow chamber. The inlet side of the flow chamber tapered from a surface area of $1.2 \mathrm{~m}^{2}$ to the $0.3 \mathrm{~m}^{2}$ of the main arena (where the inlet honeycomb was placed). Isoamyl acetate (IAA; $3 \%$ in mineral oil, Sigma-Aldrich) was released, also at $5 \mathrm{~cm} / \mathrm{s}$, through one of three odor tubes magnetically clipped on to and extending $10 \mathrm{~cm}$ in front of the turbulence grid. Each odor tube was an 18-cm-long 3-D printed horn linearly expanding from an inner diameter of 3-10 $\mathrm{mm}$ and its lower edge raised $15 \mathrm{~mm}$ above the floor (horn center at $20 \mathrm{~mm}$ off the floor). Odor tubes were located at midline and $25 \mathrm{~cm}$ lateral to midline. An air-dilution olfactometer was built to deliver odor by bubbling air through an odor vial containing 3\% IAA in mineral oil. Each odor tube isokinetically delivered either air or odor at $236 \mathrm{ml} / \mathrm{min}$. Above each odor port was a lick spout associated with that port. In the case of robot testing, LED lights were attached on top of each odor port in place of the lick spouts. All sides of the flow chamber were constructed from white acrylic and the top of the flow chamber was constructed from clear acrylic to allow for imaging during the behavioral task. A 2-inch diameter hole was cut in the base of the flow chamber directly in front of the outlet honeycomb (center at $7.5 \mathrm{~cm}$ ) along the midline (from downstream to upstream) of the chamber. This hole served as the insertion point for animals at the beginning of every trial and was immediately sealed after animal entry using a magnetic disk that was flush with the base of the flow chamber.

To increase lateral variation in the flow which in turn increases the chaotic mixing (Mehta and Bradshaw, 1979) in the SOL, we removed the inlet honeycomb, allowing ambient room air flow to add complexity in addition to the static turbulence grid (Fig. 1A; Extended Data Fig 1-1). To evaluate the effectiveness thereof, we measured odor concentration time series along the midline of the SOL at $10,30,50$, and $60 \mathrm{~cm}$ downstream from the odor tube. Three series of $120 \mathrm{~s}$ (50 samples/s) were taken at each location with the inlet honeycomb, after which the honeycomb was removed and the measurements were repeated. This entire sequence was repeated once for a total of six-time series per location per condition (Extended Data Fig. 1-1). Measurements were taken with a miniPID (Aurora Scientific) set to low gain and slow pump speed. The odor used was $50 \%$ ethanol evaporated via a stainless-steel bubbler and released isokinetically (flow conditions were identical to the experimental conditions described above). To minimally interfere with the nonturbulent chaotic airflow and ensure measurement consistency, the midline and upstream edges of the miniPID sensor body were located $15 \mathrm{~cm}$ lateral from midline and $5 \mathrm{~cm}$ downstream from the inlet tip of a 1/8-inch OD Teflon tube bent gradually at $90^{\circ}$ to suck in air in down- stream direction. A 22-gauge needle pierced the tube vertically, $2 \mathrm{~cm}$ from the tube's tip, and assured a consistent sampling height of $20 \mathrm{~mm}$. The miniPID output was directly digitized using a Syscomp 11-bit A/D board (CGM-101) and streamed to disk. The final 6000 samples of each data file were saved as MATLAB data files (https://doi.org/10.5061/dryad.zgmsbcc71) and used for analysis of complexity (MATLAB code file, available on DataDryad, https://doi.org/10.5061/dryad.zgmsbcc71). Small DC-offsets were removed. Intermittency was calculated as the fraction of time the time series was above $4.4 \%$ of the maximum average signal at $10 \mathrm{~cm}$ from the odor tube.

\section{Mouse: behavioral training}

Four adult male C57BI/6 mice aged 24-26 weeks were used. Mice were handled for 20 min each day for one week before habituation in the flow chamber. Following a week of handling with the experimenter, animals were allowed to explore the flow chamber for $30 \mathrm{~min} / \mathrm{d}$ for $5 \mathrm{~d}$. Subsequently, animals were water regulated (body weight closely monitored and maintained at $85 \%$ of original weight) and trained to associate the lick spouts with sucrose water $(100 \mathrm{mM})$ delivery. Water was dispensed free-flowing from each of the three lick spouts and animals were lick-trained until they licked from all three lick-spouts. Once lick-trained, animals were trained on a simple version of the navigation task. At the beginning of every trial, an odor plume was established from odor port $1 \mathrm{for} 30 \mathrm{~s}$, and then the animal was inserted into the arena through the 2-inch hole at the outlet end of the flow chamber. Animals were given $45 \mathrm{~s}$ to navigate to port 1 and were trained on this task for $6 \mathrm{~d}$. Animals were group housed in an environment of controlled humidity (40\%) and temperature $\left(22^{\circ} \mathrm{C}\right)$ with a $12 / 12 \mathrm{~h}$ inverted dark/light cycle with lights off at 9 A.M. Animals were tested during their dark cycle under red light. All experimental protocols were performed in accordance with protocols approved by Pierce Animal Care and Use Committee. The John B Pierce Laboratory is AAALAC accredited. These procedures are in agreement with the National Institutes of Health Guide for the Care and Use of Laboratory Animals (8th edition).

\section{Mouse: odor navigation task}

On each trial odor was released from one of three possible odor ports and isokinetic clean air was released from the other two ports. Thirty seconds was allotted for the odor plume to be established before inserting the animal. On entering the flow chamber through the 2 -inch hole at the outlet end, the animal was given $45 \mathrm{~s}$ to navigate to the odor source. If the animal reached the correct odor source, an $8-\mathrm{kHz}$ tone was played, and the animal was required to remain within the reward zone for $100 \mathrm{~ms}$ before a sucrose water reward $(100 \mathrm{mM})$ was delivered for $500 \mathrm{~ms}$. After sucrose water delivery, the animal was removed from the arena. If the animal approached an incorrect odor port or failed to reach the reward zone within the $45-\mathrm{s}$ duration, a $1-\mathrm{kHz}$ tone was played and the animal was removed from the arena. In between trials odor was turned off and the animal was 
placed in an enrichment cage for $45 \mathrm{~s}$. This amount of time was sufficient to clear any residual odor from the flow chamber. Animals were tested on 30-40 randomized trials per day with equal representation of each odor port. Animals were tested using the honeycomb condition for $14 \mathrm{~d}$ and subsequently without the honeycomb for $5 \mathrm{~d}$. Lastly, animals were tested on a no odor control paradigm.

\section{Model: geometry}

We developed in silico simulations of odor navigation in static and dynamic plumes. We refer to these simulations interchangeably as a model and simulated robot. The simulated robot makes temporally discrete sample-tosample comparisons of odor concentration at its left and right sensors as it moves through space. It consists of a virtual chassis with coordinates centered at $(x, y)$ and moves through space along a heading $\theta$ at a velocity $v$ :

$$
\begin{gathered}
x_{t+\Delta t}=x_{t}+v \Delta t \cos \theta \\
y_{t+\Delta t}=y_{t}+v \Delta t \sin \theta,
\end{gathered}
$$

where $\Delta t$ represents the update rate of the model, here $100 \mathrm{~ms}$. Velocity $v$ is $4 \mathrm{~cm} / \mathrm{s}$. The agent has a chassis radius of $\ell_{d}=8 \mathrm{~cm}$. Sensors are located at the front of the chassis with a variable intersensor distance of $\ell_{s}$. The two sensors are separated by an angle $\gamma=\arctan \left(\ell_{s} / 2 \ell_{d}\right)$. Sensor positions are given as:

$$
\begin{aligned}
& x_{L / R}=x+\sqrt{\ell_{d}^{2}+\left(\frac{\ell_{s}}{2}\right)^{2}} \cos (\theta \pm \gamma) \\
& y_{L / R}=y+\sqrt{\ell_{d}^{2}+\left(\frac{\ell_{s}}{2}\right)^{2}} \sin (\theta \pm \gamma),
\end{aligned}
$$

where $\left(x_{L}, y_{L}\right)$ is the left sensor and $\left(x_{R}, y_{R}\right)$ is the right sensor. The agent geometry is shown in Figure $3 A$.

The simulated robot engages in hierarchical navigation algorithms which begin with (1) baseline acquisition, followed by iterative (2) wall avoidance, and then (3) odordriven navigation. Both baseline acquisition and odordriven navigation require transduction of the underlying odorant concentration into a sensor signal.

\section{Model: odor signal simulation}

Odor signals at each sensor are simulated as

$$
\dot{S}_{L / R}=-k_{\text {decay }} S+C_{x, y, t} .
$$

Here, $k_{\text {decay }}$ is a rate constant set to $\ln (2) / 0.8$ s on experimental sensor half-life data (Extended Data Fig. 4-1C). $\mathrm{C}_{\mathrm{x}, \mathrm{y}, \mathrm{t}}$ represents the instantaneous concentration sampled at time $t$ from the plume dataset at the pixel position (point source) corresponding to either the left or right sensor.

Using this simple model for sensor odor signal, we may define the model's baseline acquisition and odor-driven navigation.

\section{Baseline acquisition}

Baseline acquisition is identical for both simulated algorithms. First, the simulated robot remains stationary for
$10 \mathrm{~s}$ to allow its sensors to equilibrate according to Equation 5.

After equilibration, the model remains stationary and samples from the left sensor four times over the following second. These sensor values are averaged to generate $S_{L, \text { baseline. }}$ Over the subsequent second model performs the same procedure at the right sensor to generate $S_{R \text { baseline }}$

Finally, the two baselines are averaged to obtain $S_{\text {baseline }}=\left(S_{L, \text { baseline }}+S_{R \text {,baseline }}\right) / 2$, a value which will be used in odor-driven navigation.

\section{Wall avoidance}

In each loop of the simulated robot program, the model first uses its IR sensors to determine whether it must take corrective action to avoid an arena wall. If the simulated robot's center $(x, y)$ approaches within distance $d_{\text {threshold }}$ $=10 \mathrm{~cm}$ of a wall, it takes the following corrective actions.

If the model approaches a wall from its left-hand side (i.e., if the wall is in the left IR detection radius in Fig. $3 A$, orange arc), it first turns right for $100 \mathrm{~ms}$, corresponding to a change in heading of $\sim 30^{\circ}$ to the right:

$$
\theta_{t+\Delta t}=\theta_{t}-\frac{\pi}{6}
$$

It then moves forward for $200 \mathrm{~ms}$ according to Equations $1,2$.

If the model approaches a wall from its right-hand side (i.e., if the wall is in the right IR detection radius in Fig. $3 A$, green arc), it first turns left for $100 \mathrm{~ms}$, corresponding to a change in heading of $\sim 30^{\circ}$ to the left:

$$
\theta_{t+\Delta t}=\theta_{t}+\frac{\pi}{6}
$$

It then moves forward for 200 ms according to Equations $1,2$.

If the model approaches a wall head on (i.e., if the wall is in the center IR detection radius in Fig. $3 A$, blue arc), it first turns right for $100 \mathrm{~ms}$, corresponding to a change in heading of $\sim 30^{\circ}$ to the right (Eq. 6). It then backs up for $200 \mathrm{~ms}$ according to Equations $1,2(\mathrm{v}=-4 \mathrm{~cm} / \mathrm{s}$ to reverse course).

Following any of the above scenarios, the model remains stationary for $300 \mathrm{~ms}$ to allow the sensors to equilibrate.

\section{Model: odor-driven navigation}

If no wall is encountered in a loop of the robot code, it engages in odor-driven navigation. Here, model behavior varies depending on whether algorithm A or B is implemented.

In algorithm A, the sensors are queried and one of three alternatives is selected based on current sensor values in order of precedence:

1. If the value $\left(S_{L}-S_{\text {baseline }}\right)-\left(S_{R}-S_{\text {baseline }}\right)>S_{\text {threshold }}$, $\left(S_{\text {threshold }}=0.03\right)$, the model turns left for $100 \mathrm{~ms}$ according to Equation 7. It then moves forward for $200 \mathrm{~ms}$ according to Equations 1, 2.

2. If the value $\left(S_{R}-S_{\text {baseline }}\right)-\left(S_{L}-S_{\text {baseline }}\right)>S_{\text {threshold }}$, the model turns right for $100 \mathrm{~ms}$ according to Equa- 
tion 6. It then moves forward for $200 \mathrm{~ms}$ according to Equations 1, 2.

3. If neither 1 nor 2 occur, the model goes straight for $200 \mathrm{~ms}$ according to Equations 1, 2.

Following any of the above three scenarios, the model remains stationary for $300 \mathrm{~ms}$ to allow the sensors to equilibrate.

In algorithm B, memory of the previous average odor sample is retained. The sensors are queried and the temporal difference in average concentration values is computed:

$$
\begin{gathered}
\Delta \bar{C}=\frac{1}{2}\left[\left(\left(S_{L}-S_{\text {threshold }}\right)+\left(S_{R}-S_{\text {threshold }}\right)\right)_{t}-\left(\left(S_{L}-\right.\right.\right. \\
\left.\left.\left.S_{\text {threshold }}\right)+\left(S_{R}-S_{\text {threshold }}\right)\right)_{t-\Delta t}\right] .
\end{gathered}
$$

Using this value and the sensor values, one of four alternatives is selected based on current sensor values in order of precedence:

1. If $\Delta \bar{C}>\mathrm{S}_{\text {threshold }} / 4$, the model goes straight for 200 $\mathrm{ms}$ according to Equations 1, 2.

2. If the value $\left(S_{L}-S_{\text {baseline }}\right)-\left(S_{R}-S_{\text {baseline }}\right)>S_{\text {threshold }} / 2$, the model turns left for $100 \mathrm{~ms}$ according to Equation 7. It then moves forward for $200 \mathrm{~ms}$ according to Equations 1, 2.

3. If the value $\left(S_{R}-S_{\text {baseline }}\right)-\left(S_{L}-S_{\text {baseline }}\right)>S_{\text {threshold }} / 2$, the model turns right for $100 \mathrm{~ms}$ according to Equation 6 . It then moves forward for $200 \mathrm{~ms}$ according to Equations 1, 2.

4. If none of the above are true, the model proceeds forward for $200 \mathrm{~ms}$ according to Equations 1, 2.

Following any of the above three scenarios, the model remains stationary for $300 \mathrm{~ms}$ to allow the sensors to equilibrate. For algorithm $\mathrm{A}$ and $\mathrm{B}$, the simulated robots are allotted $75 \mathrm{~s}$ to find the odor source.

\section{Model: plume data}

Four minutes of near-surface acetone planar laserinduced fluorescence (PLIF) plume data from Connor et al. (2018) was used as input for these models (11282017_10cms_bounded.h5,/dataset7). The above models are deterministic. If they are synchronized with the first frame of the plume dataset, they will always generate the same trajectory. To simulate "random" complexity, each model evaluation initialized the plume dataset at a randomly chosen frame between 1 and 3600; the 4-min dataset was then allowed to loop continuously until the simulation concluded (Movie 1, Movie 2).

To study the effect of a non-turbulent low chaos environment on model performance, we took the time average of the $4 \mathrm{~min}$ of plume data to generate a smooth static environment (Fig. 3C).

\section{Robot: design}

We purchased and modified an Arduino robot (Extended Data Fig. 4-1A; Arduino robot, code: A000078, Arduino was purchased from Robotshop). The Arduino robot comes equipped with a control board (on top) with a control pad to turn ON/OFF the robot, an LCD screen to read the sensory data, a compass, a processor, and

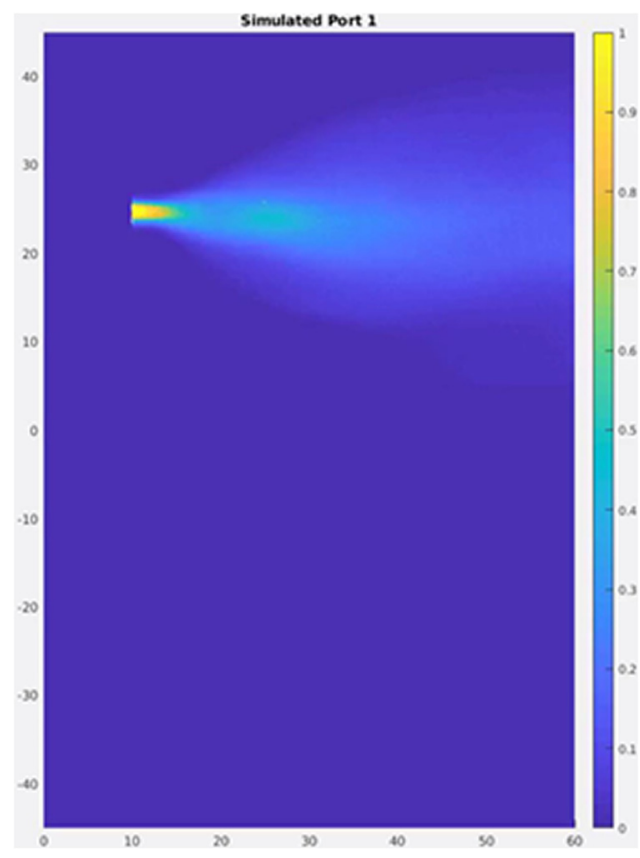

Movie 1. In silico dynamic plume released from corner port. Video played at $10 \mathrm{~Hz}$ (first $10 \mathrm{~s}$ shown). [View online]

analog/digital inputs to attach a variety of sensors. Additionally, the robot contains a motor board (on bottom) with two wheels for movement, a processor, ON/OFF switch, a power jack $(9 \mathrm{~V})$, an interboard connector, a reset button for troubleshooting and a USB port to connect the robot with any device or computer. The robot can be programmed using Arduino software (Arduino Software IDE, 1.8.5 version). The same bare robot platform was also used for gas source localization by Ali Yeon et al. (2018).

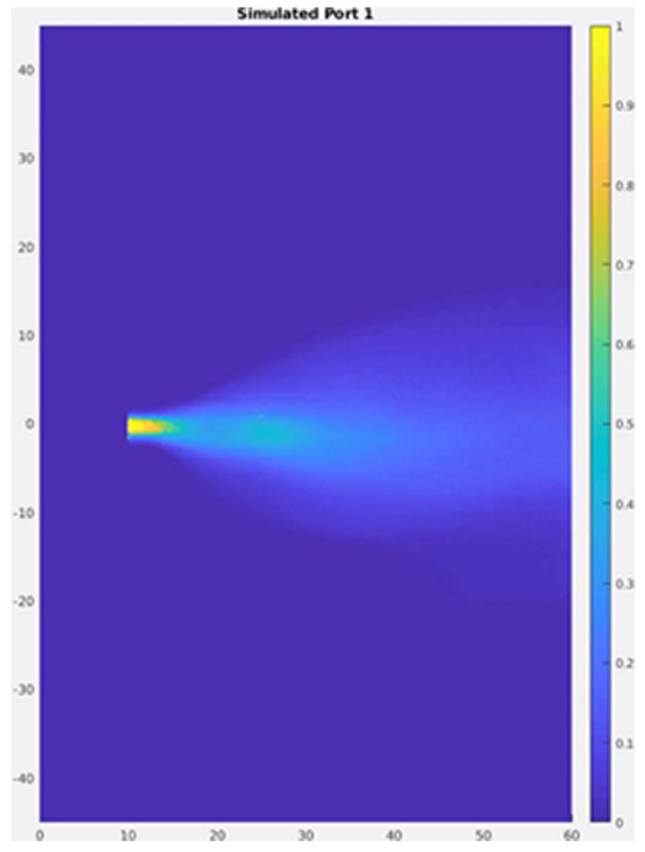

Movie 2. In silico dynamic plume released from center port. Video played at $10 \mathrm{~Hz}$ (first $10 \mathrm{~s}$ shown). [View online] 
To power the hardware, we mounted three step-down buck DC-DC converters (DROK, 3A) connected to three dual lithium ion battery (Samsung 18650, 3.6 V, 3000 $\mathrm{mAh}$ ) holders connected in parallel, providing $3.0 \mathrm{~V}$ (fans), $5.0 \mathrm{~V}$ (robot), and $6.5 \mathrm{~V}$ (gas sensors). Two (left and right) gas sensors [DFRobot, Analog Gas Sensor, MQ-2 (DFRO$\mathrm{BOT}$ ) an Arduino package based on MQ-2 gas sensor by the Hanwei Electronics Co. (hwsensor)] with a high sensitivity to detecting alcohol (and a variety of volatile organic compounds such as LPG, methane, hydrogen and smoke) were installed on the robot (Extended Data Fig. 4-1A). The gas sensor's tin oxide layer on the aluminum oxide ceramic tube is heated by a nickel-chromium alloy coil and has an odor concentration-dependent resistance, suitable to detect a range of concentrations of gasses at constant temperature and humidity. To increase the response speed (Extended Data Fig. 4-1C), both gas sensors were modified by drilling a hole in the PC-board behind the sensor and attaching a gas sensor fan $(10 \times 10$ $\times 5 \mathrm{~mm}$, UF3A5-100, Sunon, run at $3.0 \mathrm{~V}, 0.9 \mathrm{l} / \mathrm{min}$ ) to suck in air from the front to back, and removing the front of the perforated metal grid. The sensors were powered at $6.5 \mathrm{~V}$ instead of the standard $5 \mathrm{~V}$. Also, we designed a pair of 3-D printed holders, rods and clamps, to incorporate the gas sensors at the top of the robot to allow adjustment of the distance between them and their angle in the horizontal plane. In addition, we added an analog ambient light sensor (DFRobot, V2, SKU:DFR0026) mounted at the front of the robot at the base of a frontally oriented cone and three IR-based proximity sensors (Sharp, GP2Y0A41SK0F; Extended Data Fig. 4-1A) at the center, left and right sides on the top board. Codes run on the Arduino robot are in accordance with the algorithm $A$ and $\mathrm{B}$ described for the in silico model. These algorithms have been made available DataDryad (https://doi.org/10.5061/ dryad.zgmsbcc71).

Response dynamics of the gas sensors were evaluated with a custom Arduino code reading the sensor voltage 100 times per second. Sensors were stimulated by rapidly manually passing an alcohol-saturated cotton swab from left to right at 0.5 inches from the frontal plane of the sensors $\sim 15.2 \mathrm{~s}$ after starting a trial. The baseline reading (mean of first 100 samples) was subtracted and response maxima were normalized to 1 . Individual responses were time-aligned to the peak and smoothed with a threesample running average. Reported data are averages of 2-10 trials, ignoring several trials with more than one peak and/or non-exponential decay.

\section{Robot: odor navigation task}

As in the mouse odor navigation task, at the beginning of every trial, odor was released from one of three odor ports and isokinetic air flow was released from remaining two ports. Odor plume was established for $10 \mathrm{~s}$ before the beginning of the trial. The real robot, as opposed to the simulated in silico robot described above, was allotted 75 $s$ to navigate to the odor source. The robot was tested on odor navigation to all three odor ports from varying start angles from a center start position along the midline of the outlet end of the flow chamber. For odor port 1 (right-most odor port), the robot was tested at start angles of $90^{\circ}$, $135^{\circ}$, and $180^{\circ}$, for odor port 2 (center odor port), the robot was tested at start angles of $135^{\circ}, 180^{\circ}$, and $225^{\circ}$, for odor port 3 (left-most odor port) the robot was tested at start angles of $180^{\circ}, 225^{\circ}$, and $270^{\circ}$ (Fig. 4A). For each of these start angles, the robot was tested once with sensor angles of $0^{\circ}$ and $45^{\circ}$ as well as with sensor distances of 8 and $16 \mathrm{~cm}$. Both Code A and Code B were tested in the presence of the honeycomb and Code $B$ was tested without the honeycomb. The robot was also tested from a corner start position where it was located at a $270^{\circ}$ angle at the right-most corner of the outlet end of the flow chamber. This start position was tested using active odor port 2. For this start position the robot was tested once with sensor angles of $0^{\circ}$ and $45^{\circ}$ as well as with sensor distances of 8 and $16 \mathrm{~cm}$. Both Code A and Code B were tested with and without the honeycomb for this start position. The robot was tested for 10 trials for every condition.

\section{Code accessibility}

All codes have been made available on DataDryad (https:// doi.org/10.5061/dryad.zgmsbcc71). Additionally, all codes are in Extended Data 1. Included are MATLAB and Arduino codes to generate the center and corner odor plumes (file names: odorFun_plume_center.m, odorFun_plume_corner.m), test the in silico-simulated robot using Code A and Code B (filenames: SimRobot_test_A.m, SimRobot_test_B.m), and to test the in silico model with replicates (filenames: run_model_A_replicates.m, run_model_B_replicates.m). Additionally, this folder contains two Arduino codes for robot navigation (file names: Robot_CodeA.ino, Robot_CodeB.ino). These files were run on Windows 10.

\section{Behavioral tracking and data analysis}

All behavioral tracking, for both the mouse and robot, was conducted using Noldus behavioral tracking system (EthoVision XT, version 10.1, Noldus Information Technology) and trajectories were further analyzed using MATLAB (R2018a, The MathWorks). GraphPad Prism (version 7; GraphPad Software, Inc.) was used to generate graphs and conduct statistical analyses. For all group comparisons, statistical tests were corrected for multiple comparison using a Bonferroni correction when appropriate (Table 1). Mouse data represents the average for each mouse across all days for the given condition. Robot data represents the average across 10 trials per condition. Model data represents the average across 20 simulations. All data are represented as mean \pm SEM.

\section{Results}

\section{Mice successfully locate odor source within a non- turbulent chaotic flow chamber}

To test mouse navigation within an airborne odor plume, we built a $1 \times 1 \times 0.3 \mathrm{~m}$ flow chamber behavioral arena based on that used by Connor et al. (2018). We introduced two honeycombs on either end to laminarize the airflow established by a vacuum at the outlet end. To generate a controlled complex odor plume within this flow 


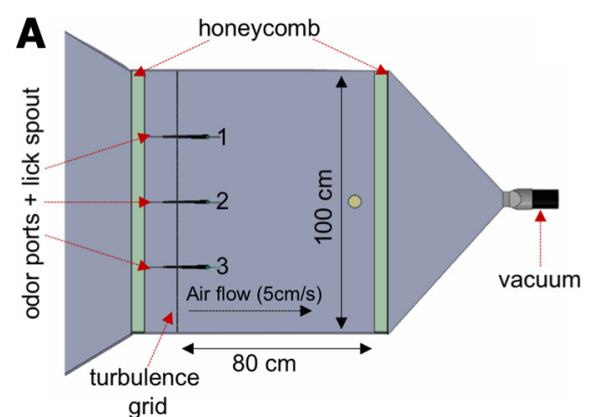

C

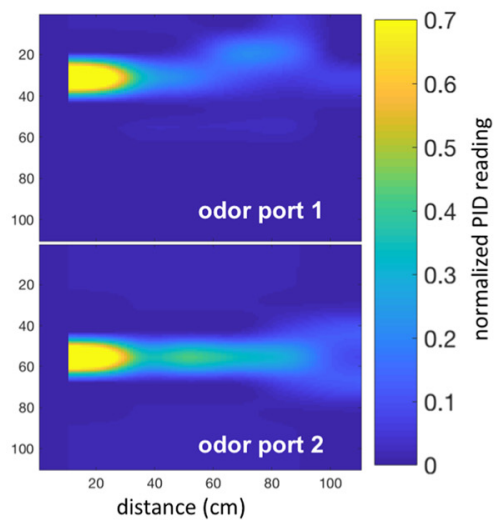

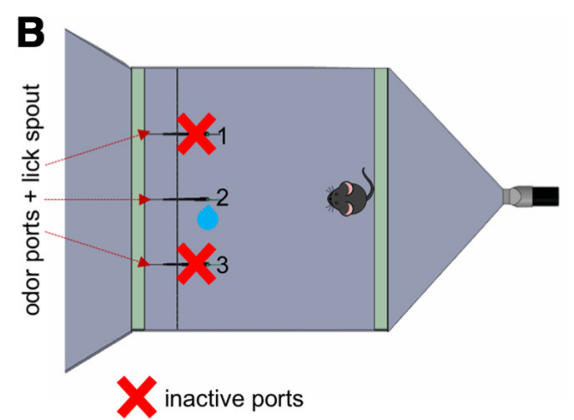

D

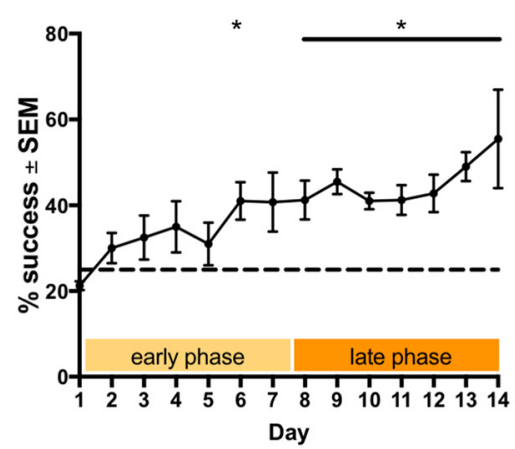

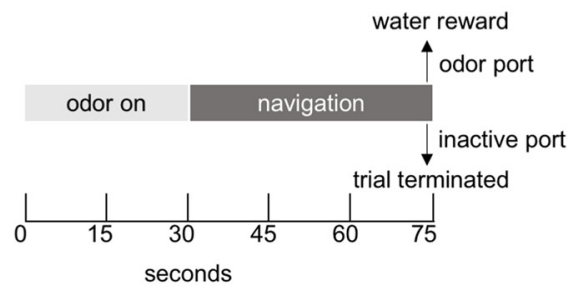

E

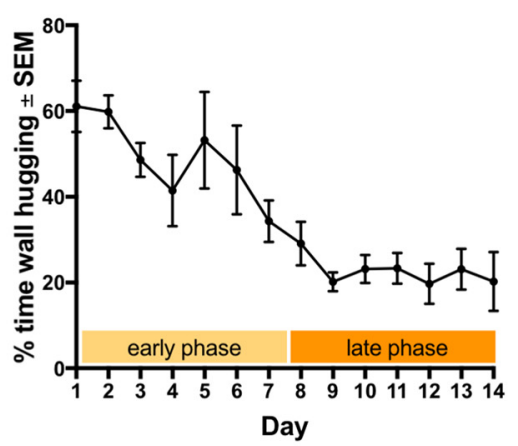

Figure 1. Mouse odor-navigation task. $\boldsymbol{A}$, Flow chamber used to conduct behavioral assay. Chamber is flanked by two honeycombs and on the inlet side, a turbulence grid $10 \mathrm{~cm}$ in front of the honeycomb. Three odor ports and lick spouts are spaced along inlet side and vacuum is used to establish air flow $(5 \mathrm{~cm} / \mathrm{s})$. $\boldsymbol{B}$. Mouse is rewarded for navigating to the port releasing odor (port two) and trial is terminated early if animal navigates to incorrect port (left). Trial structure includes a 30-s period to establish plume before animal enters chamber and given $45 \mathrm{~s}$ to navigate (right). $\boldsymbol{C}$, miniPID readings of odor concentration from odor ports 1 and 2 (time averaged and normalized to maximum reading which occurs at the odor source). $\boldsymbol{D}$, Performance (\% successful trials in a given session) of mice over testing days. Performance is broken up into an early phase (first $7 \mathrm{~d}$ ) and a late phase (last $7 \mathrm{~d}$ ). Plot shows mean performance \pm SEM, $n=4$ mice. $E$, Percentage of time spent hugging the chamber wall, defined as within $5 \mathrm{~cm}$ of behavioral arena wall, over testing days. Plot shows mean $\%$ time spent wall hugging $\pm \mathrm{SEM}, n=4$ mice. See also Extended Data Figure 1-1. $* p<0.05$.

chamber, we inserted a turbulence grid in front of the honeycomb at the inlet end (Fig. 1A). A flow rate of $5 \mathrm{~cm} / \mathrm{s}$ was established within the flow chamber. For the purposes of this study, we refer to this flow chamber as a SOL. Three odor ports at the inlet end of the flow chamber released odor, generating plumes. We measured the time averaged concentration of odor across the flow chamber within each of the three plumes using a miniature photoionization detector, miniPID (Fig. 1C).

We trained a group of mice on a task to navigate to the source of these airborne odor plumes within the SOL. On any given trial, an odor plume was established from one of the three odor ports for $30 \mathrm{~s}$ before the insertion of the animal into the behavioral arena. The task structure required water-regulated mice to locate an odor port releasing IAA ( $3 \%$ in mineral oil) within $45 \mathrm{~s}$ to receive a sucrose water reward from an adjacent lick spout (Fig. 1B; Movie 3). Other studies aimed at understanding rodent navigation within airborne odor plumes have found that with experience animals preferentially use a localization strategy in which they serially explore all possible odor source locations, showing a shift away from using solely odorbased cues (Bhattacharyya and Bhalla, 2015; Gire et al., 2016). To ensure that the mice in this study relied only on odor information, we terminated trials when the mouse reached one of three odor ports, providing water reward only if the odor-releasing port (i.e., not the two clean air-releasing ports) was reached. This behavioral design incentivizes mice to make a decision regarding odor source location, rather than testing all possible sources.

Before being tested on this task, animals were trained to associate the localization of an odor port releasing odor with delivery of a sucrose water reward. Animals were able to learn the task following a $6 \mathrm{~d}$ of this training and performed consistently above chance starting the eight day of testing (Fig. 1D; one-tailed two-sample $t$ test with Holm-Sidak correction for multiple comparisons, $p=$

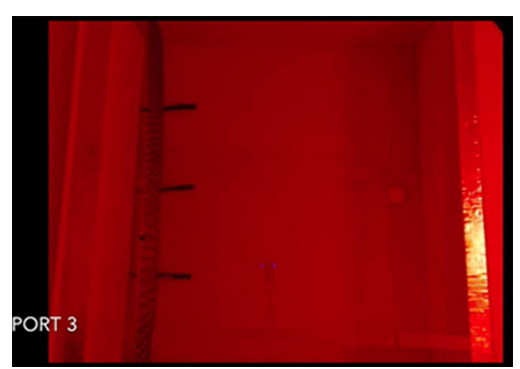

Movie 3. Mouse navigation to airborne odor source. In first trial animal, odor port 3 is releasing odor. In second trial, odor port 2 is releasing odor. Video recorded and played back at $15 \mathrm{~Hz}$. [View online] 
0.047 for day $6, p=0.047$ for day $8, p=0.0026$ for day $9, p=0.0013$ for day $10, p=0.018$ for day $11, p=0.033$ for day $12, p=0.0026$ for day $13, p=0.047$ for day 14 , $n=4$ mice). Thus, the testing days were classified into two phases of $7 \mathrm{~d}$ each, the early phase and the late phase. Thigmotaxis (wall-hugging) behavior indicates an anxiety-like state in mice. Mice decreased the percentage of the 45-s trial spent engaging in wall-hugging behavior over time (Fig. 1E; paired one-tailed $t$ test, late phase vs early phase difference: $-27.03 \pm 2.79, p=0.0012, n=4$ mice $^{\mathrm{a}}$ ).

\section{Mouse performance remains robust with increased complexity but shows a shift in strategy}

To test the effect of increased complexity on odor localization performance, we removed the honeycomb at the inlet side of the flow chamber (Extended Data Fig. 1-1). This allows for the introduction of ambient air complexity into the behavioral arena in addition to that caused by the turbulence grid. We refer to this odor environment as "non-turbulent chaotic" as well as "complex." When comparing the two environments, we refer to the honeycomb condition interchangeably with "low-complexity" and the no honeycomb condition with "high-complexity" environments. The SD s of the 2-min odor concentration time series at each midline downstream location (six repeats each) were all significantly increased by roughly two- to four-fold $(3.9,2.3,1.8$, and 2.1 times the SD with inlet honeycomb at $10,30,50$, and $60 \mathrm{~cm}$ downstream from the odor tube, respectively). The SD normalized by mean odor concentration was also significantly increased at 10 and $30 \mathrm{~cm}$ from the odor tube by 4.0 - and 1.9 -fold, respectively. Note that instrument noise contribution to the SD was negligible.

Animals perform at a significantly higher \% success in the late phase when compared to the early phase and show no change in performance between the late phase and no honeycomb condition (Fig. $2 A$; paired $t$ test onetailed, late phase vs early phase difference $=11.65 \pm$ $3.1 \%, p=0.016^{\mathrm{b}}$, paired $t$ test two-tailed, no honeycomb vs late phase difference $=-1.92 \pm 2.74 \%, p=0.53^{\mathrm{c}}, n=$ 4 mice). This shows a significant improvement of performance over time in the same odor environment and that with increased odor plume complexity animals show consistent task performance. Additionally, no difference in performance is seen across ports between the late phase and the no honeycomb condition, although there was a small effect of port number (Extended Data Fig. 2-1A; two-way ANOVA, main effect of plume complexity, $p=$ 0.8 , main effect of port $=0.039, n=4$ mice). This effect of port number may be because the animals were licktrained on odor port 1 (although post hoc $t$ tests with Bonferroni correction for multiple comparisons do not reveal a significant difference between ports, port 1 vs port 2 difference: $26.2 \pm 10.23 \%, p=0.0917^{d}$, port 1 vs port 3 difference: $28.35 \pm 10.23 \%, p=0.065^{\mathrm{e}}$, port 2 vs port 3 difference: $37.67 \pm 10.23 \%, p>0.99^{f}, n=4$ mice). To ensure that animals were using odor information for this task, we tested them on a set of $\sim 30$ trials without odor between the late phase and no honeycomb condi- tion. Animals performed at chance levels without odor and their performance was significantly lower than that during the late phase or no honeycomb phase (Fig. 2A; paired $t$ test one-tailed, no odor vs late phase difference: $-31.32 \pm$ $6.24, p=0.0076^{9}$, no odor vs no honeycomb difference: $-29.4 \pm 5.22, p=0.0055^{\mathrm{h}}, n=4$ mice).

We recorded behavior during trials using a camera placed above the flow chamber and imaged through the transparent lid of the behavioral arena. We found that on successful trials, the distance and time to the target odor port decreases between the early and late phase (Fig. $2 B-D$; paired $t$ test two-tailed, distance to target of late phase vs early phase difference: $-60.79 \pm 16.8 \mathrm{~cm}, p=$ $0.036^{i}$, time to target of early phase vs late phase difference: $-4.6 \pm 0.73 \mathrm{~s}, p=0.008^{\mathrm{j}}, n=4$ mice), showing that animals are taking shorter and faster routes to the correct odor port over time. Additionally, the early phase shows a significant negative linear trend of time to correct odor port over time, whereas the late phase does not show a significant decline. Thus, their behavior has stabilized when entering into the late phase (Fig. $2 D$; linear regression, $R^{2}=0.62$ early phase, $p=0.0357, R^{2}=0.006$ late phase, $p=0.71, n=4$ mice).

We measured several parameters associated with the animals' behavior during the trial, as the level of odor plume complexity could affect the path taken and parameters modulated during the animals' trajectories. We found that when the honeycomb was removed and complexity was increased, the distance to the target on successful trials remained the same as the late phase, but the time to the target significantly decreased (Fig. 2B,C; paired $t$ test two-tailed, distance to target no honeycomb vs late phase difference: $-3.52 \pm 7.05 \mathrm{~cm}, p=0.65^{\mathrm{k}}$, time to target no honeycomb vs late phase difference: $-1.99 \pm$ $0.57 \mathrm{~s}, p=0.039^{1}, n=4$ mice). Additionally, the animals traveled at a higher velocity when navigating a more chaotic plume (Fig. 2E,G; paired $t$ test two-tailed, no honeycomb vs late phase difference: $8.044 \pm 2.37 \mathrm{~cm} / \mathrm{s}$, $p=0.043^{\mathrm{m}}, n=4$ mice).

Casting involving lateral full-body or head movement during odor-based navigation is a behavioral strategy that has been extensively characterized and found to be conserved across several species (Vickers, 2000; Grasso, 2001). Invertebrates including moths, flies, and cockroaches implement this zig-zagging behavior when localizing odor within an airborne odor plume, particularly when attempting to reacquire the odor stream (David et al., 1983; Kennedy, 1983; Baker and Haynes, 1987; Kuenen and Cardé, 1994; Grasso, 2001; Cardé and Willis, 2008; Gomez-Marin et al., 2011; van Breugel and Dickinson, 2014). Additionally, mammals, including both rodents and humans, display lateral head movements when tracking odor trails (Porter et al., 2007; Khan et al., 2012; Catania, 2013). Here we measured "casting" using two parameters. The first is the path curvature as measured by the absolute total sum of turning angles during a trial. Animals did not display any difference in turning behavior between the late phase and no honeycomb condition (Fig. 2F; Extended Data Fig. 2-1B; paired $t$ test two-tailed, no honeycomb vs late phase difference: $-27.19 \pm 13.39^{\circ}, p$ 
A
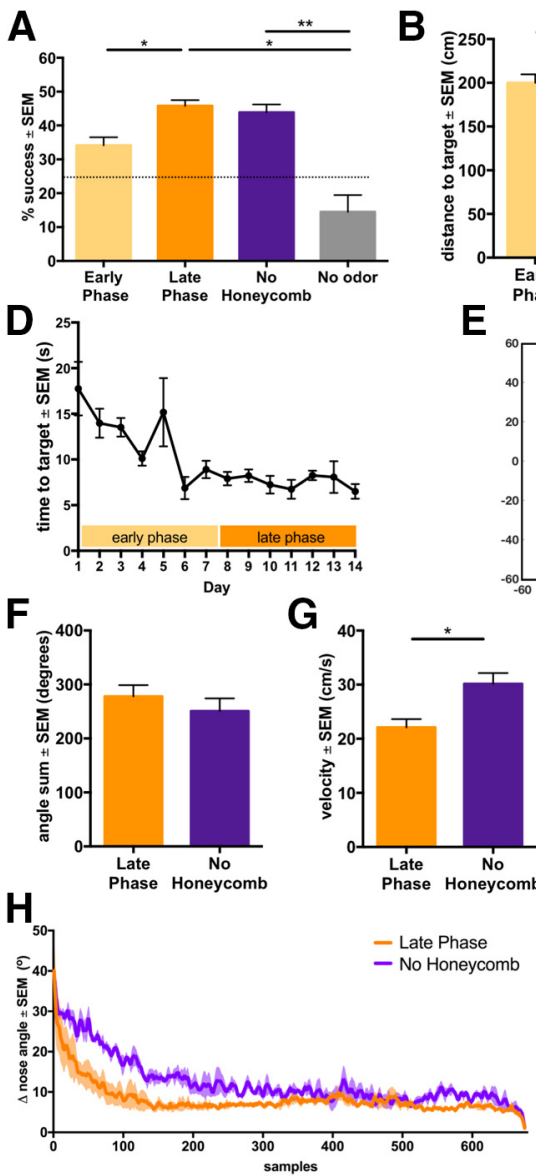

G

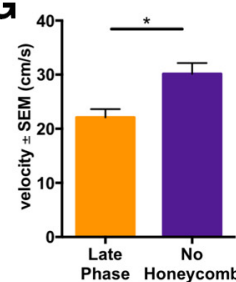

B

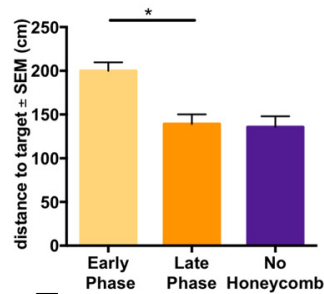

E

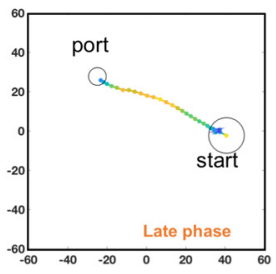

C
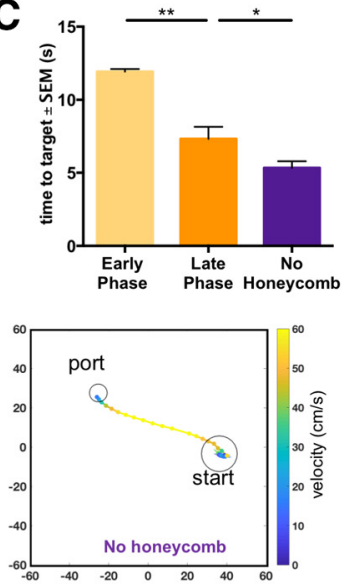
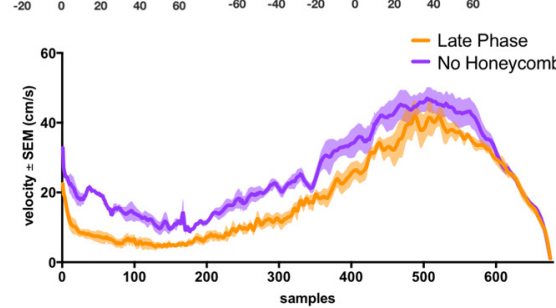

I
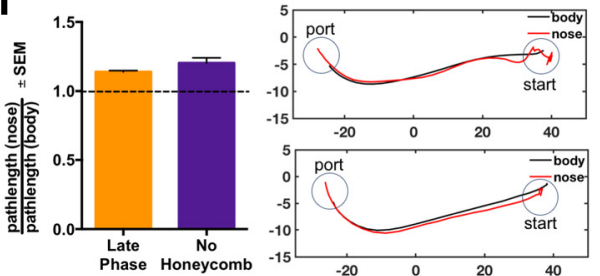

Figure 2. Mice change navigation behavior with increased experience and odor environment complexity. $\boldsymbol{A}$, Performance (average \% successful trials over sessions) across testing phases. Mice are tested on a no-odor condition in addition to the phases with a honeycomb and condition without a honeycomb. Chance level performance is $25 \%$ as animals have three ports as options and are not required to choose an odor port on trials. $\boldsymbol{B}$, Pathlength to target odor port on successful trials. $\boldsymbol{C}$, Time to target odor port on successful trials. $\boldsymbol{D}$, Time to target on successful trials over testing days. $\boldsymbol{E}$, Example traces of successful navigation from the late phase and no honeycomb phase. Traces are color scaled based on velocity. $\boldsymbol{F}$, Total angle sum of trajectories of late phase and no honeycomb condition. Total angle sum is calculated by using the total sum of angles on turns from frame-to-frame. $\mathbf{G}$, Velocity on successful trials of late phase and honeycomb condition (left). Velocity over the course of successful trajectories resampled to 675 frames (right). $\boldsymbol{H}$, Change in nose angle per frame $(15 \mathrm{~Hz}$ ) over the course of successful trajectories resampled to 675 frames (left). Change in nose angle on successful trials of late phase and no honeycomb condition (right). I, Ratio of path distance based on nose to path distance based on center of body (left). Example trajectories with ratios of 1.35 (top) and 1.08 (bottom). All plots show mean \pm SEM, $n=4$ mice. See also Extended Data Figure 2-1. $* p<0.05, * * p<0.01$.

$=0.14^{\mathrm{n}}$, two-way ANOVA, total angle sum main effect of plume complexity, $p=0.92$, total angle sum main effect of port number, $p=0.63 ; n=4$ mice). Average total sum of turning angles for both conditions are below $360^{\circ}$, and thus mouse turning behavior remains below a full rotation during navigation, suggesting minimal full-body casting. This lack of casting behavior is in alignment with previous observations in rodents navigating in odor plumes (Bhattacharyya and Bhalla, 2015; Gire et al., 2016). The second form of casting measured was the change in nose angle, thereby measuring sweeps in head movement during odor localization. We found that mice show modest changes in nose angle which are slightly higher when the chaotic nature of the odor plume is increased (Fig. $2 \mathrm{H}$; paired $t$ test two-tailed, no honeycomb vs late phase difference: $2.94 \pm 0.83^{\circ}, p=0.04^{\circ}, n=4$ mice). Additionally, the ratio of the trial pathlength as measured by the nose position to that measured by the body position shows that nose pathlength is greater than body pathlength (Fig. 2/; one-sample $t$ test, $\mu=1$, late phase mean: $1.14 \pm 0.004, p<0.0001^{p}$, no honeycomb mean: $1.20 \pm$ $0.02, p=0.0016^{q}, n=4$ mice). Thus, this suggests that mice do not display lateral body movements, but do exhibit sweeping movements with their head during odor plume navigation. However, these head movements appear to be limited to the initial phase of olfactory search behavior (Fig. $2 \mathrm{H}$ ).

Interestingly, trajectories from one test session show few differences between the late phase and no honeycomb condition (Fig. 5A). Additionally, animals' path linearity, as measured by the fraction of distance of a straight-line path to that of the actual path, did not vary across rewarded ports, showing consistency across tested plumes (Extended Data Fig. 2-1C; two-way 
ANOVA, linearity main effect of plume complexity, $p=$ 0.81 , linearity main effect of port number, $p=0.9, n=4$ mice). Overall, these results suggest that increased odor plume complexity does not affect odor navigation performance. However, animals do alter their strategy when navigating a more chaotic plume, where a faster speed may be beneficial for odor localization, whereas modulating parameters that affect trajectory structure may not be as important.

\section{Model-based odor navigation}

To compare mouse odor navigation with simple odor localization algorithms, we developed an in silicosimulated robot. The simulated robot has two odor sensors, with a separation distance that can be varied, and can make comparisons between the odor signals at the left and right sensor. It has a virtual frame and moves through a virtual odor plume with a heading $\theta$. If the simulated robot approaches the wall of the virtual arena, it will take corrective measures to reorient toward the open arena (Fig. 3A). We tested this in silico model in a virtual SOL with a center and corner port, analogous to that in which we tested the mice (Fig. $3 B$ ). We tested the simulated robot navigation starting at the center of the arena with start angles varying from $90^{\circ}$ to $270^{\circ}$ at $3.6^{\circ}$ increments. Acetone PLIF data were used as the odor plume input for the virtual arena, obtained from Connor et al. (2018). To assess the effect of odor plume complexity on the behavior of our model, we tested the simulated robot using either a static odor plume (i.e., the average of 4 min of odor plume data) or using a dynamic odor plume with real-time fluctuations (Fig. 3C).

We created two navigational algorithms to test in silico odor localization. These algorithms were designed to incorporate a minimal interpretation of stereo smell while, in one case, also incorporating features to resolve the fluctuating nature of our odor plume. For both algorithms a baseline reading is collected for each sensor as the average of four readings over $1 \mathrm{~s}$. These two baselines are then averaged to be used for odor-based navigation. In the first algorithm, which we refer to as Code $A$, if the difference between the instantaneous sensor reading at the left sensor and the right sensor, both corrected for the baseline reading, is greater than the threshold (described in Materials and Methods), the model turns left and moves forward for a subsequent reading. If the difference between the right sensor and the left sensor reading, corrected for the baseline, is greater than the threshold, the model turns right and advances. If neither of these conditions are true, the model moves forward.

The most basic model implemented in a robotics approach aimed at odor plume tracking is one in which the robot with a pair of chemical sensors simply moves in the direction of higher concentration. However, this approach may be limited due to the previously described dynamic nature of odor plumes in which the robot can at one moment sense odor that quickly disappears while remaining stationary (Sandini et al., 1993; Kazadi et al., 2000; Lilienthal and Duckett, 2004; Ishida et al., 2012). Models that rely on averaging several frames on odor intake before determining movement may be more successful at determining concentration gradients (Ishida et al., 2001). Using this logic, we created Code B. In this algorithm, if the difference between the average (of the readings of the two sensors) across two time points is greater than a threshold, the model will move forward, as this indicates the simulated robot is moving up the concentration gradient. Otherwise, Code B defaults to the same rules described for Code A.

\section{In silico-simulated robot navigation is affected by increased plume complexity}

As previously mentioned, stereo smell is important for odor navigation in both mammals and invertebrates. The distance between olfactory sensors may play a role in the ability of an animal to accurately detect an odor plume and locate the source. We tested the simulated robot in both the static and dynamic odor plumes with two sensor separation distances, 16 and $8 \mathrm{~cm}$. Model Code A performs at a significantly lower success rate in the presence of increased plume complexity at an 8-cm sensor separation distance regardless of active odor port position (Fig. 3D; Extended Data Fig. 3-6; two-tailed $t$ test center port Code A 8-cm static vs center port Code A $8-\mathrm{cm}$ dynamic difference: $8.37 \pm 1.1 \%, p<0.0001^{r}$, two-tailed $t$ test corner port Code A 8-cm static vs corner port Code A 8-cm dynamic difference: $3.91 \pm 0.84 \%, p<0.0001^{\mathrm{s}}$, $n=20$ simulations). Additionally, Code A at $8 \mathrm{~cm}$ shows a decrease in trajectory linearity as an average and across starting angles when the plume complexity increases, suggesting that with increased complexity, paths become more winding (Fig. 3E; Extended Data Fig. 3-6; two-tailed $t$ test center port Code A 8-cm static vs center port Code A 8-cm dynamic difference: $0.065 \pm 0.007, p<0.0001^{\mathrm{t}}$, two-tailed $t$ test corner port Code A 8-cm static vs corner port Code A 8-cm dynamic difference: $0.023 \pm 0.0035, p$ $<0.0001^{\mathrm{u}}, n=20$ simulations). Model Code B shows a significant decrease in performance with increased plume complexity at a 16-cm sensor separation distance with a center odor plume and an 8-cm sensor separation distance regardless of plume position (Fig. 3D; Extended Data Fig. 3-6; two-tailed $t$ test center port Code B 8-cm static vs center port Code B 8-cm dynamic difference: $20.11 \pm 1.1 \%, p<0.0001^{v}$, center port Code B 16-cm static vs center port Code B 16-cm dynamic difference: $3.70 \pm 1.1 \%, p=0.011^{\mathrm{w}}$, corner port Code B 8-cm static vs corner port Code B 8-cm dynamic difference: $5.54 \pm$ $0.84 \%, p<0.0001^{x}, n=20$ simulations). Data from both codes show that at an $8-\mathrm{cm}$ sensor separation distance, algorithms are more susceptible to a decrease in performance due to increased odor plume complexity.

Additionally, linearity as an average and across starting angles for Code B decreases with increasing plume complexity, indicating that with either sensor separation distance, paths become less linear with increased complexity (Fig. 3E; Extended Data Fig. 3-6; two-tailed $t$ test center port Code B 8-cm honeycomb vs center port Code B 8-cm no honeycomb difference: $0.15 \pm 0.007, p<$ $0.0001^{y}$, center port Code B 16-cm honeycomb vs center port Code B 16-cm no honeycomb difference: $0.03 \pm$ 
A

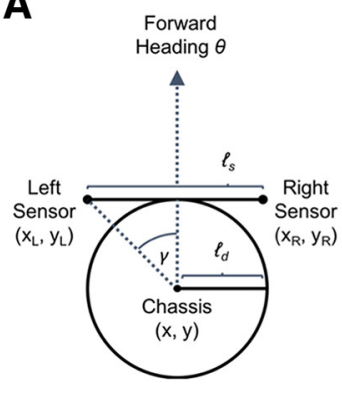

C Dynamic Plume, Sample Frame

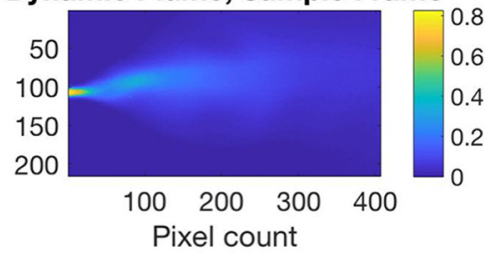

D

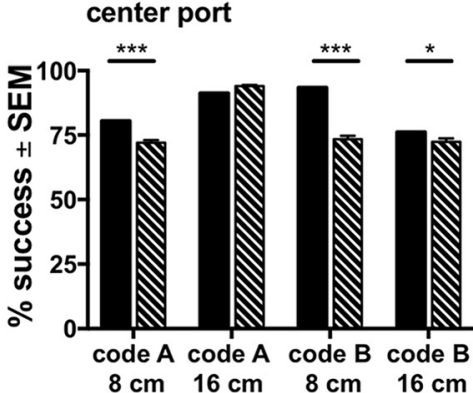

E

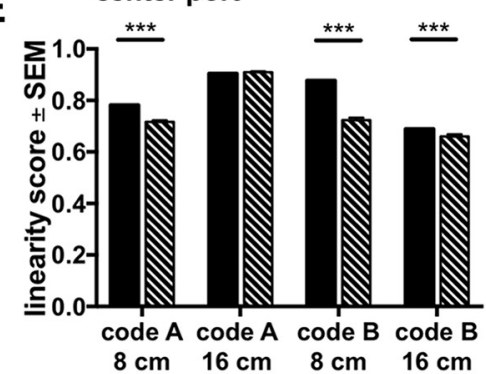

B

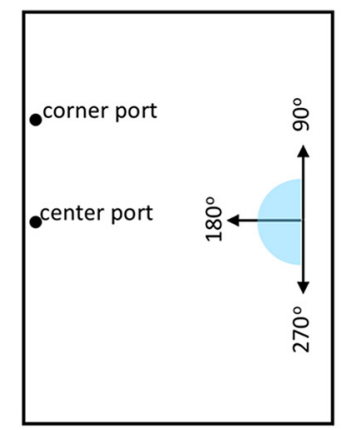

Time-Averaged Static Plume

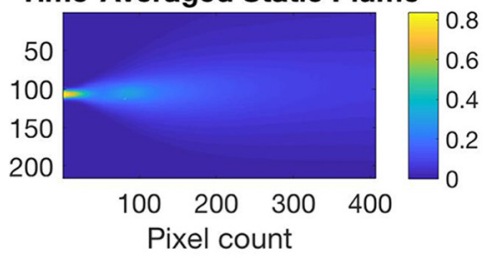

corner port

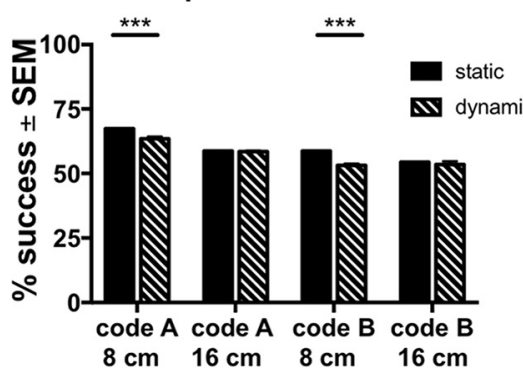

corner port

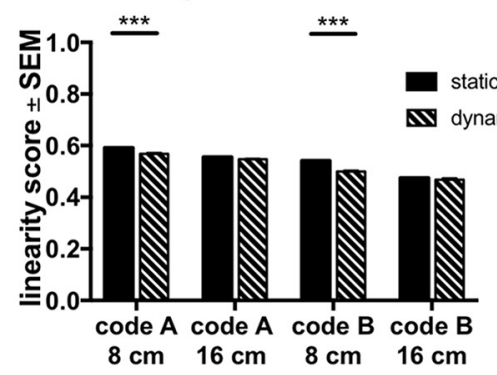

Figure 3. In silico models show decreased performance with increased odor environment complexity. $\boldsymbol{A}$, Model virtual chassis moves through space with a heading, $\theta$. Two sensors are separated at a distance $\ell_{s}$ and an angle $\gamma$ (left). If the center of the model reaches $\mathrm{d}_{\text {threshold }}=10 \mathrm{~cm}$ of the wall, the model will take corrective measures (right; described in Materials and Methods). $\boldsymbol{B}$, Model is tested at angles ranging from $90^{\circ}$ to $270^{\circ}$ with a start position in the center of the arena. Model is tested on two plumes, one originating from a center port and one from a corner port. $\boldsymbol{C}$, Sample frame depicting instantaneous concentration of the dynamic plume normalized to odor source (left), and an image of the stationary concentration gradient in static plume normalized to odor source (right). $\boldsymbol{D}$, Performance (average \% success of all start angles \pm SEM) across code and sensor distance for center target port (left) and corner target port (right); $n=20$ simulations. $\boldsymbol{E}$, Linearity score (calculated as the ratio of the Euclidean distance between start point and end point of trajectory and the actual pathlength) across code and sensor distance for center target port (left) and corner target port (right). Plot shows mean linearity score \pm SEM, $n=20$ simulations. See also Extended Data Figures 3-1, 3-2, 3-3, 3-4, 3-5, and 3-6. $* p<$ $0.05, * * p<0.01, * * * p<0.001$.

$0.007, p=0.0006^{z}$, corner port Code B 8-cm honeycomb vs corner port Code B 8-cm no honeycomb difference: $0.042 \pm 0.003, p<0.0001^{\text {aa }}, n=20$ simulations). Trajectories within the static odor plume are deterministic as there is a fixed odor plume gradient to climb, whereas there was variation in the paths within the dynamic plume, as expected (Extended Data Figs. 3-1, 3-2, 3-3, 3-4, 3-6;
Movies 4, 5, 6, 7, 8, 9, 10, and 11). Interestingly, both the success and linearity of Code $B$ at an $8-\mathrm{cm}$ separation distance in the dynamic plume shows periodicity where the success and linearity decrease and rise every $30^{\circ}$ of starting angles (Extended Data Fig. 3-6). This periodicity may be attributed to the $30^{\circ}$ turn angle implemented in silico and if the simulated robot is capable of rotating to 


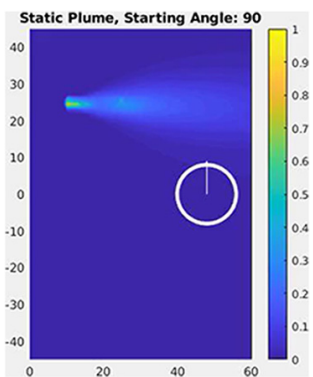

Movie 4. In silico model navigation of static odor plume released from corner odor port using Code A. Video recorded at $10 \mathrm{~Hz}$ and played back at $60 \mathrm{~Hz}$. [View online]

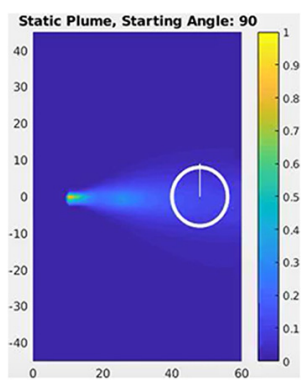

Movie 5. In silico model navigation of static odor plume released from center odor port using Code A. Video recorded at $10 \mathrm{~Hz}$ and played back at $60 \mathrm{~Hz}$. [View online]

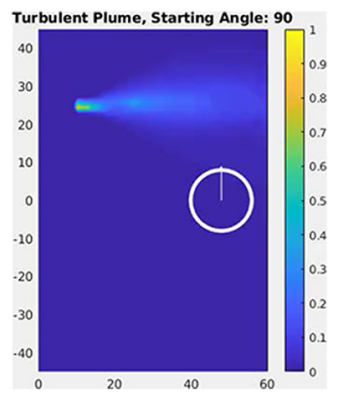

Movie 6. In silico model navigation of dynamic odor plume released from corner odor port using Code A. Video recorded at $10 \mathrm{~Hz}$ and played back at $60 \mathrm{~Hz}$. [View online]

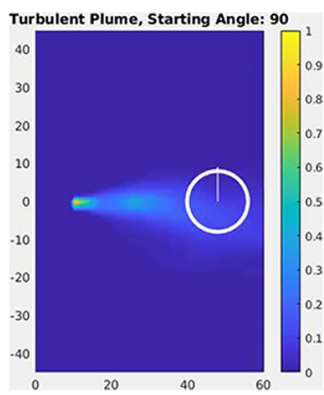

Movie 7. In silico model navigation of dynamic odor plume released from center odor port using Code $\mathrm{A}$. Video recorded at $10 \mathrm{~Hz}$ and played back at $60 \mathrm{~Hz}$. [View online]

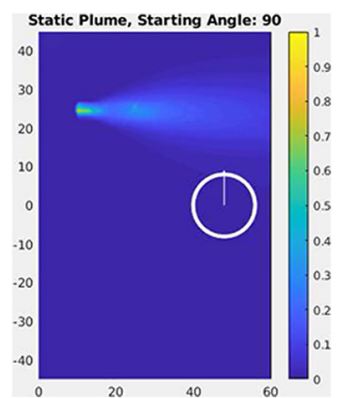

Movie 8. In silico model navigation of static odor plume released from corner odor port using code $\mathrm{B}$. Video recorded at $10 \mathrm{~Hz}$ and played back at $60 \mathrm{~Hz}$. [View online]

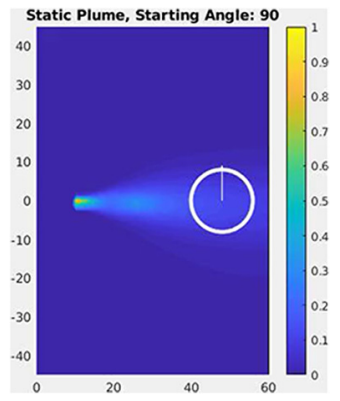

Movie 9. In silico model navigation of static odor plume released from center odor port using code $\mathrm{B}$. Video recorded at $10 \mathrm{~Hz}$ and played back at $60 \mathrm{~Hz}$. [View online]

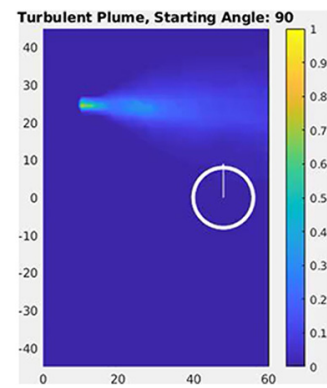

Movie 10. In silico model navigation of dynamic odor plume released from corner odor port using code $\mathrm{B}$. Video recorded at $10 \mathrm{~Hz}$ and played back at $60 \mathrm{~Hz}$. [View online]

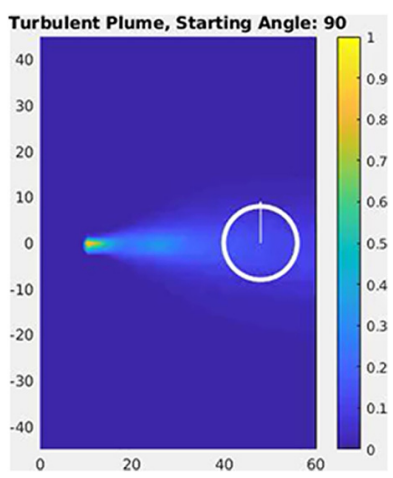

Movie 11. In silico model navigation of dynamic odor plume released from center odor port using code $\mathrm{B}$. Video recorded at $10 \mathrm{~Hz}$ and played back at $60 \mathrm{~Hz}$. [View online] 
$180^{\circ}$ (facing the odor source) using the increment, it will ultimately be more successful and have a straighter path.

When comparing performance across codes, in the static condition, Code A had a significantly lower \% success than Code B at an 8-cm sensor separation distance, however Code B performed significantly worse than Code $A$ at a $16-\mathrm{cm}$ sensor separation distance, showing the interaction between code and sensor separation distance (Fig. 3D; two-tailed $t$ test center port Code A 8-cm static vs Code B 8-cm static difference: $-13.04 \pm 1.1 \%, p<$ 0.0001 , center port Code A 16-cm static vs Code B 16-cm static difference: $15.22 \pm 1.1 \%, p<0.0001^{\mathrm{bb}}, n=20$ simulations). In the dynamic condition, just as in the static condition, Code A performs significantly better than Code $\mathrm{B}$ at a 16-cm sensor separation distance (Fig. 3D; Code A $16-\mathrm{cm}$ turbulent vs Code B $16-\mathrm{cm}$ turbulent difference: $21.63 \pm 1.1 \%, p<0.0001^{\mathrm{cc}}, n=20$ simulations). Together, these findings suggest that with a small sensor separation distance Code B is more successful, however at a larger sensor separation distance Code $A$ is more successful.

Difference in trajectories between static and dynamic conditions can be observed in Figure 5C. Our simulated robot was tested using data collected in the SOL at the same starting position as the mice, therefore we can directly compare performance between the two. Model Code A overall performs with a higher \% success than the mice, but there is no significant difference between performance of model code $B$ and the mice [Extended Data Fig. 5-1A, left; two-tailed $t$ test low-complexity mouse vs Code A difference: $-25.68 \pm 8.74 \%, p=0.043^{\text {dd }}$, highcomplexity mouse vs Code A difference: $-25.38 \pm 8.74 \%$, $p=0.048^{\mathrm{ee}}$, low-complexity mouse vs Code B difference: $-21.68 \pm 8.74 \%, p=0.12^{\mathrm{ff}}$, high-complexity mouse vs Code B difference: $-16.63 \pm 8.74 \%, p=0.42^{\mathrm{gg}}, n=4$ mice, $n=4$ sessions for each model condition (one session for per combination of sensor distance and target odor port)]. Additionally, mice locate the odor source on successful trials significantly faster than both codes [Extended Data Fig. 5-1B, two-tailed $t$ test low-complexity mouse vs Code A difference: $-33.75 \pm 3.63 \mathrm{~s}, p<$ $0.0001^{\text {hh }}$, high-complexity mouse vs Code A difference: $-36.59 \pm 3.63 \mathrm{~s}, p<0.0001^{\mathrm{ii}}$, low-complexity mouse vs Code B difference: $-35.25 \pm 3.63, p<0.0001^{\mathrm{jj}}$, highcomplexity mouse vs Code B difference: $-39.01 \pm 3.63 \mathrm{~s}$, $p<0.0001^{\mathrm{kk}}, n=4$ mice, $n=4$ sessions for each model condition (one session for per combination of sensor distance and target odor port)]. These findings show that although the Code A outperforms a mouse in terms of \% success for the low and high plume complexity conditions, both codes show a decrease in within code performance in the presence of increased complexity, a behavioral shift not seen in mice.

\section{Arduino-based robot shows decrease in performance with increased odor plume complexity}

To test how our in silico models perform in a real flow chamber, we tested an Arduino-based robot using Code A and Code B in the previously described SOL behavioral arena. We modified the arena to replace lick spouts with
LEDs associated with each odor port which were detected by light sensors on the robot to identify if an odor port had been approached. The Arduino-based robot was equipped with optimized gas sensors attached to a fan that actively sucked air through the sensors. In addition, we attached proximity sensors to avoid contact with the walls of the flow chamber. The gas sensors were optimized for response speed by removing the front of steel mesh cap surrounding the front of the sensor, drilling a hole through the pc-board behind the sensor and fitting a small fan on the back of the hole (Extended Data Fig. 4-1A). The responsiveness of the sensor was improved by an order of magnitude: time from stimulus onset (i.e., the first time the signal crosses $2 \%$ of peak amplitude) to $75 \%$ of peak (t75 O) was $0.67 \mathrm{~s}$ in the unmodified sensor but reduced to $0.07 \mathrm{~s}$ when modified, being $1.13 \mathrm{~s}$ and $0.11 \mathrm{~s}$ (t100 O) to reach peak value, respectively (Extended Data Fig. 4-1C). Decay time from peak to $50 \%$ of peak (t50 off) was reduced from 2.41 to $0.47 \mathrm{~s}$, and to $25 \%$ of peak (t25 off) from 4.96 to $2.14 \mathrm{~s}$, respectively. The distance between these gas sensors could be varied, as well as the angle at which they were oriented.

We tested the robot starting on the midline of the outlet end of the flow chamber for direct comparison with mouse and in silico model behavior. We used six different starting angles with varying active odor ports based on starting angle (Materials and Methods; Fig. 4A). At this starting position, we tested the robot using Code $A$ and Code $B$ with the honeycomb as well as Code B without the honeycomb. Additionally, we recorded behavior at an alternate start position, which cannot be directly compared to the mouse behavior, in which the start angle of the robot was $270^{\circ}$ at the far-right corner of the outlet end of the chamber. In this condition the center port was used for plume generation (Extended Data Fig. 4-1D). At this start position, we tested the robot using both Code $A$ and Code B with and without the honeycomb. At both starting positions we tested the robot with sensor separation distances of 8 and $16 \mathrm{~cm}$ and sensor angles of $0^{\circ}$, parallel with the front of the robot, and $45^{\circ}$. Additionally, we tested the robot using $70 \%$ ethanol instead of IAA, used with mice, to obtain robust odor readings from the robot's gas sensors. The task structure for the robot odor-based navigation was nearly identical to that of the mouse; however, the robot was allotted $75 \mathrm{~s}$ to reach the odor source.

We studied how the behavior of the robot changed when tested with the two algorithms in the presence of increased complexity by removing the honeycomb at the inlet side of the flow chamber, the exact conditions we tested on the mice. Code A showed a decrease in performance at the corner start position when the honeycomb was removed and Code $B$ show a significant decrease in $\%$ success with increased complexity at both start positions (Fig. 4B, left, C; Extended Data Fig. 4-1E, left; paired two-tailed $t$ test, corner start Code $A$ no honeycomb vs Code $\mathrm{A}$ with honeycomb difference: $-62.5 \pm 11.09 \%, p=$ $0.011^{\prime \prime}$, center start Code B no honeycomb vs Code B with honeycomb difference: $-19.64 \pm 2.43 \%, p=$ $0.004^{\mathrm{mm}}$, corner start Code B no honeycomb vs Code B 
A

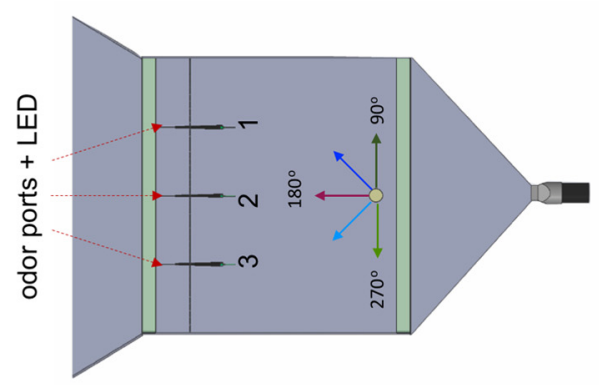

B

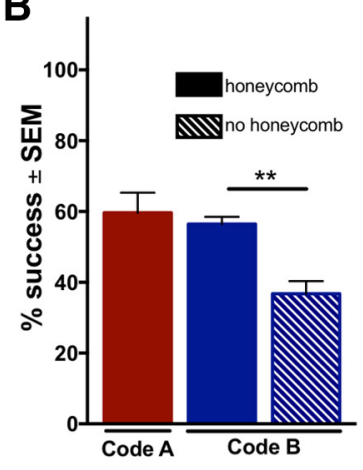

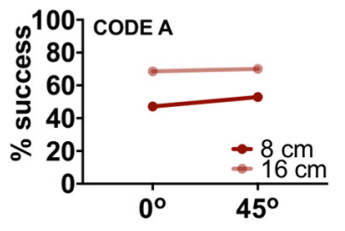

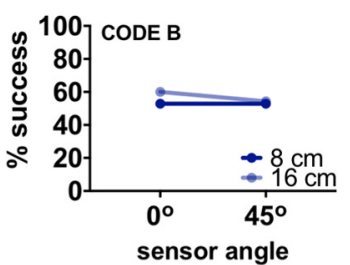

C
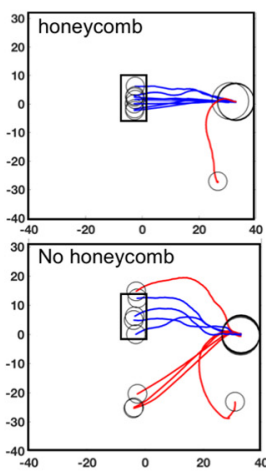

D

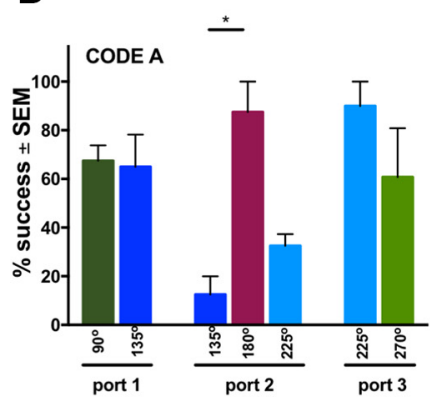

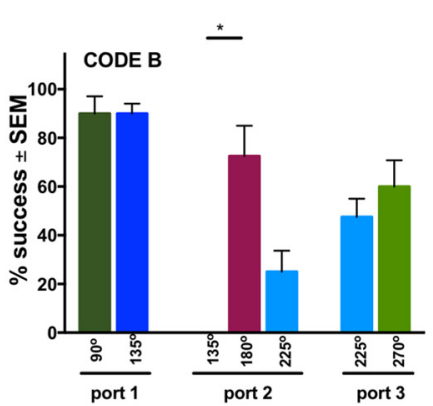

E
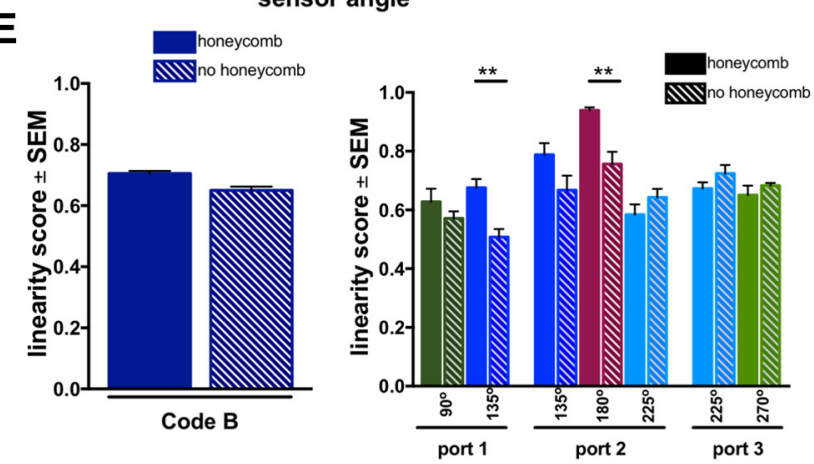

Figure 4. Arduino-based robot navigation varies based on start position and odor environment complexity. $\boldsymbol{A}$, Robot odor navigation flow chamber, modifications to the SOL. Solid arrows represent five starting angles. Odor ports were coupled to LED lights detected by sensors on the robot (indicated by dotted red arrows). B, Performance (average \% successful trials over 8 and $16 \mathrm{~cm}$ and $0^{\circ}$ and $45^{\circ}$ gas sensor distance and angles, respectively) across codes (left). Performance based on gas sensor distance and angle for the honeycomb condition (right). $\boldsymbol{C}$, Example trajectories from $180^{\circ}$ (magenta) starting position in $\boldsymbol{A}$ for honeycomb and no honeycomb condition. $\boldsymbol{D}$, Performance (average \% successful trials over 8 and $16 \mathrm{~cm}$ and $0^{\circ}$ and $45^{\circ}$ gas sensor distance and angles, respectively) with the honeycomb based on starting angle and rewarded port for Code A (left) and Code B (right). Bars are color coded and labeled according to the starting angles in $\boldsymbol{A}$. $\boldsymbol{E}$, Robot overall linearity score with honeycomb and without honeycomb using Code B. Plot shows data combined over sensor angle and sensor distance for each odor environment condition (left). Linearity score across starting angles and target ports with and without the honeycomb. All plots show mean \pm SEM, $n=4$ sessions. See also Extended Data Figure 4-1. $* p<0.05, * * p<0.01$.

with honeycomb difference: $-47.5 \pm 6.29 \%, p=$ $0.0048^{\mathrm{nn}}, n=4$ sessions). Additionally, when implementing Code A with the honeycomb, the robot shows a higher success rate at a greater sensor separation for both sensor angles at a center start position and at a $0^{\circ}$ sensor angle at a corner start position (Fig. 4B, right; Extended Data Fig. 4-1E, right). A larger sensor separation distance may be beneficial for the robot navigation using Code $A$ because larger spatial differences in the concentration gradient can be detected. This finding is in line with that of the in silico model.

Performance of the robot also varies based on starting angle. When the center port is active, the robot performs at a higher \% success when oriented directly toward the source than when angled $45^{\circ}$ away from the source (Fig. $4 D$, one-way ANOVA port 2, Code A effect of start angle, $p=0.0021$, two-tailed $t$ test, Code A $180^{\circ}$ vs Code A $135^{\circ}$ difference: $75 \pm 10.41 \%, p=0.017^{\circ \circ}$, Code $A 180^{\circ}$ vs Code A $225^{\circ}$ difference: $55 \pm 12.58 \%, p=0.067^{\mathrm{pp}}$, Code $B$ effect of start angle, $p=0.0055$, Code B $180^{\circ}$ vs Code B $135^{\circ}$ difference: $72.5 \pm 12.5 \%, p=0.031^{\text {q9 }}$, Code B $180^{\circ}$ vs Code B $225^{\circ}$ difference: $47.5 \pm 13.77 \%, p=$ $0.12^{\text {rr }}, n=4$ sessions). Increased complexity in the odor environment also caused a change in the path character- istics of the robot. For Code $B$, the path linearity decreased for several start angles (Fig. 4E; two-tailed $t$ test port $1135^{\circ}$ with honeycomb vs port $1135^{\circ}$ no honeycomb difference: $0.17 \pm 0.046, p=0.0063^{\text {ss }}$, two-way ANOVA port 2, interaction between starting angle and plume complexity, $p=0.028$, port $2180^{\circ}$ with honeycomb vs port $2180^{\circ}$ without honeycomb difference: 0.18 $\pm 0.051, p=0.0068^{\mathrm{tt}}, n=4$ simulations).

When compared to in silico paths, Arduino-tested Code $B$ trajectories are significantly more linear than in silicotested Code B trajectories in both low-complexity and high-complexity environments (Extended Data Fig. 5-1D; two-tailed $t$ test low-complexity robot Code $\mathrm{B}$ vs model Code B difference: $0.22 \pm 0.071, p=0.031^{\text {uu }}$, highcomplexity robot Code B vs model Code B difference: $\left.0.25 \pm 0.071, p=0.01^{v v}\right)$. This discrepancy maybe be due to the wide range of starting angles tested for each odor port using in silico algorithms. Additionally, there is no significant difference between performance of Code B in silico and in the real flow chamber using the Arduino robot (Extended Data Fig. 5-1A, left; two-tailed $t$ test lowcomplexity robot Code B vs model Code B difference: $-11.07 \pm 8.74 \%, p>0.99^{w w}$, high-complexity robot Code $B$ vs model Code B difference: $-23.74 \pm 8.74 \%, p=$ 
A
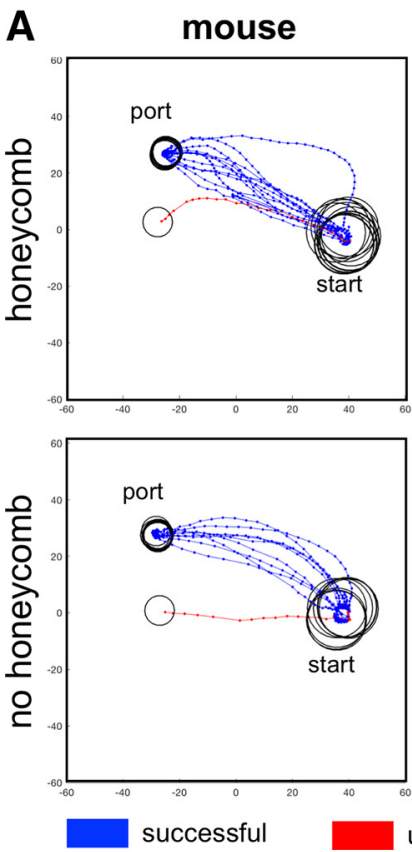

B
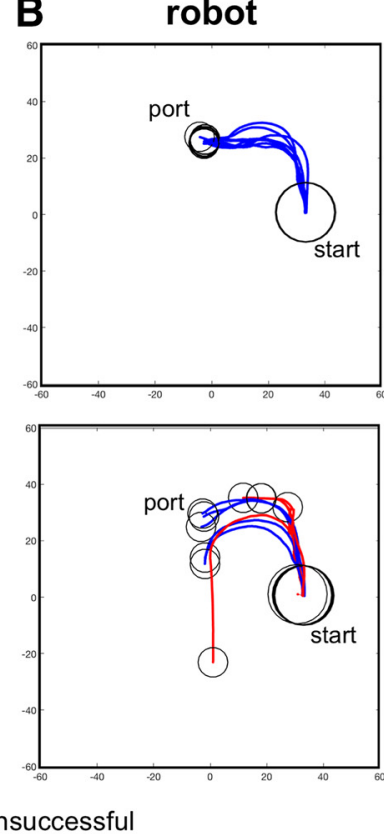
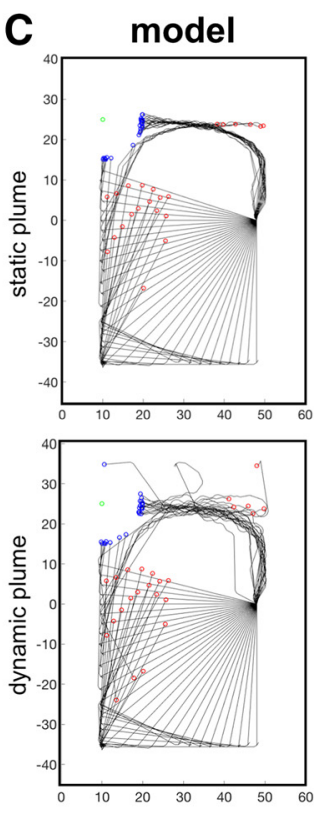

Figure 5. Mouse, robot, and in silico navigation trajectories. A, Mouse trajectories show consistency with increased odor environment complexity. $\boldsymbol{B}$, Robot trajectories show decreased success on trials for the same testing conditions with increased odor plume complexity, Code B, sensor distance: $8 \mathrm{~cm}$, sensor angle: $0^{\circ} . \mathbf{C}$, In silico trajectories (50 trials with start angles ranging from $90^{\circ}$ to $270^{\circ}$ ) show increased unsuccessful trials for the same testing conditions with increased complexity, Code B, sensor distance: $8 \mathrm{~cm}$. See also Extended Data Figure 5-1.

$0.07^{\mathrm{xx}}, n=4$ sessions). When model performance is determined selectively for the same start angles as tested on the robot, there is no significant difference between performance with low plume complexity between the robot and the model. Additionally, this subset of model data shows that the robot and the model show similar decreases in performance when the honeycomb is removed (Extended Data Fig. 5-1A, right; two-tailed $t$ test lowcomplexity robot Code B vs model Code B difference: $-34.16 \pm 10.18 \%, p=0.091$, high-complexity robot Code $B$ vs model Code B difference: $-49.58 \pm 9.48 \%, p<$ 0.0001 , one-tailed $t$ test robot Code B high vs lowcomplexity difference: $-40.41 \pm 11.01 \%, p=0.028$, one-tailed $t$ test model Code $B$ high vs low-complexity difference: $-30.83 \pm 11.72 \%, p<0.0001, n=4$ conditions). Just as in the in silico model, the robot using Code $B$ takes a significantly longer amount of time to reach the odor source on successful trials and has a significantly lower velocity when compared to mice (Extended Data Fig. 5-1B; two-tailed $t$ test low-complexity mouse vs robot Code B time to target difference: $-36.49 \pm 3.63 \mathrm{~s}, p<$ $0.0001^{\mathrm{yy}}$, high-complexity mouse vs Code B time to target difference: $-41.55 \pm 3.63 \mathrm{~s}, p<0.0001^{\mathrm{zz}}$, low-complexity mouse vs robot Code B velocity difference: $20.93 \pm 1.44$ $\mathrm{cm} / \mathrm{s}, p<0.0001^{\text {aaa }}$, high-complexity mouse vs robot Code B velocity difference: $29.06 \pm 1.44 \mathrm{~cm} / \mathrm{s}, p<$ $0.0001^{\mathrm{bbb}}, n=4$ mice, $n=4$ sessions). Difference in trajectories between static and dynamic conditions can be observed in Figure $5 B$, Movies 12, 13, 14, 15, 16, 17, 18 , and 19. Overall, our results show that when algorithms selected using in silico testing are implemented in a real flow chamber, our findings are comparable to those in silico. Additionally, just as in our in silico model, robot navigation shows a dramatic decrease in performance with increased odor plume complexity that is not observed in mouse behavior.

\section{Discussion}

Information from highly dynamic airborne odor plumes drives critical survival behaviors in animals. Variation in properties of these plumes can cause significant changes in odor-localization strategies (Mafra-Neto and Cardé, 1994; Keller and Weissburg, 2004). Here we compare the differences in odor navigation performance with increased plume complexity in mice, an in silico-simulated model, and an Arduino-based robot. We found that all three were able to successfully navigate to airborne odor sources. However, mouse performance remained robust when complexity within the plume was increased whereas in silico model and robot performance dropped. Thus, the simple binaral and temporal algorithms implemented in the model and robot are sufficient for successful navigation in a low-complexity environment, but these strategies are susceptible to declined performance when the plume becomes more chaotic. If not directly compared to mammalian odor-localization performance, these shortcomings in model performance may not have been effectively identified. With the goal of identifying minimalist biologically plausible rules that can capture animal navigation behavior, we highlight the importance of testing candidate algorithms in the same odor environment as behaving animals.

An increase in the chaotic nature of an odor environment has varying effects on odor source localization from 


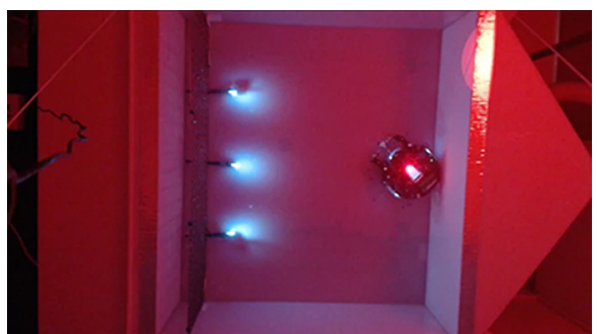

Movie 12. Arduino robot navigation to airborne odor source with honeycomb using Code $A$ with sensors at angle $0^{\circ}$ and distance $8 \mathrm{~cm}$. Odor source is middle port (port 2) and start angle is indicated in lower left corner $\left(135^{\circ}, 190^{\circ}\right.$, and $\left.225^{\circ}\right)$. Video recorded at $30 \mathrm{~Hz}$ and played back at $90 \mathrm{~Hz}$. [View online]

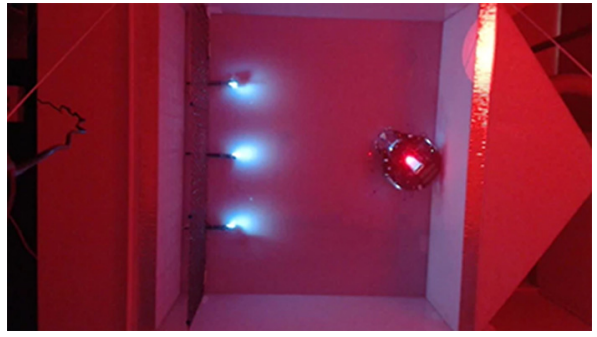

Movie 13. Arduino robot navigation to airborne odor source with honeycomb using Code A with sensors at angle $45^{\circ}$ and distance $8 \mathrm{~cm}$. Odor source is middle port (port 2) and start angle is indicated in lower left corner $\left(135^{\circ}, 190^{\circ}\right.$, and $\left.225^{\circ}\right)$. Video recorded at $30 \mathrm{~Hz}$ and played back at $90 \mathrm{~Hz}$. [View online]

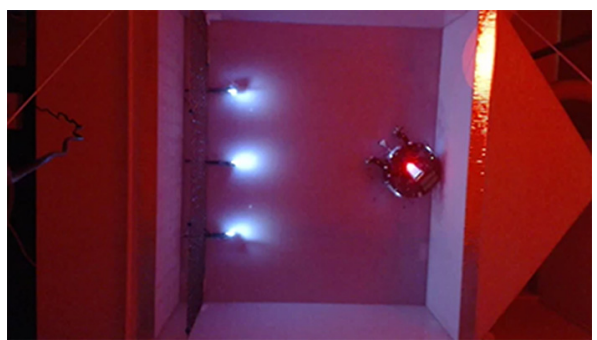

Movie 14. Arduino robot navigation to airborne odor source with honeycomb using Code A with sensors at angle $0^{\circ}$ and distance $16 \mathrm{~cm}$. Odor source is middle port (port 2) and start angle is indicated in lower left corner $\left(135^{\circ}, 190^{\circ}\right.$, and $\left.225^{\circ}\right)$. Video recorded at $30 \mathrm{~Hz}$ and played back at $90 \mathrm{~Hz}$. [View online]

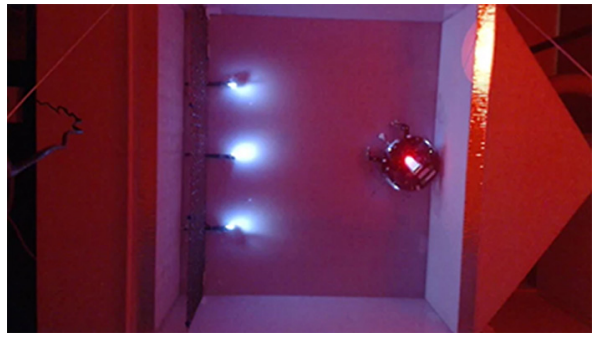

Movie 15. Arduino robot navigation to airborne odor source with honeycomb using Code A with sensors at angle $45^{\circ}$ and distance $16 \mathrm{~cm}$. Odor source is middle port (port 2) and start angle is indicated in lower left corner $\left(135^{\circ}, 190^{\circ}\right.$, and $\left.225^{\circ}\right)$. Video recorded at $30 \mathrm{~Hz}$ and played back at $90 \mathrm{~Hz}$. [View online]

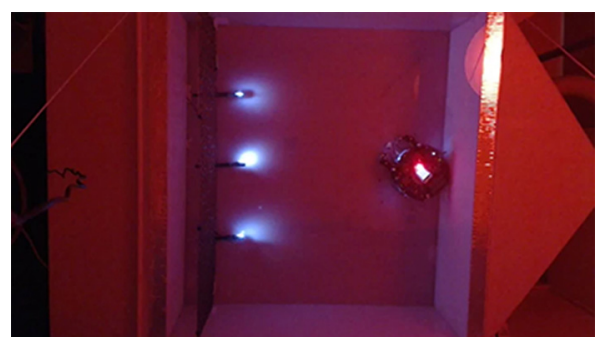

Movie 16. Arduino robot navigation to airborne odor source using code $B$ with sensors at angle $0^{\circ}$ and distance $8 \mathrm{~cm}$. Odor source is middle port (port 2), start angle is indicated in lower left corner $\left(135^{\circ}, 190^{\circ}\right.$, and $\left.225^{\circ}\right)$, condition indicated in lower left corner (honeycomb and no honeycomb). Video recorded at 30 $\mathrm{Hz}$ and played back at $90 \mathrm{~Hz}$. [View online]

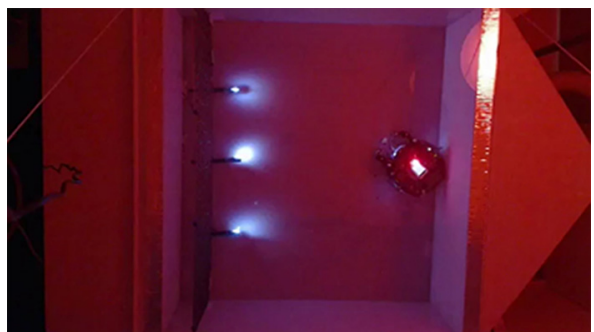

Movie 17. Arduino robot navigation to airborne odor source using code B with sensors at angle $45^{\circ}$ and distance $8 \mathrm{~cm}$. Odor source is middle port (port 2), start angle is indicated in lower left corner $\left(135^{\circ}, 190^{\circ}\right.$, and $\left.225^{\circ}\right)$, condition indicated in lower left corner (honeycomb and no honeycomb). Video recorded at 30 $\mathrm{Hz}$ and played back at $90 \mathrm{~Hz}$. [View online]

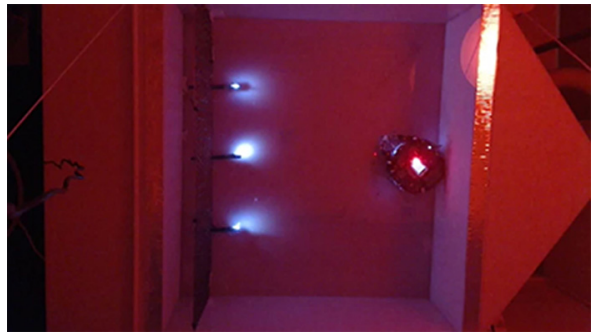

Movie 18. Arduino robot navigation to airborne odor source using code $B$ with sensors at angle $0^{\circ}$ and distance $16 \mathrm{~cm}$. Odor source is middle port (port 2), start angle is indicated in lower left corner $\left(135^{\circ}, 190^{\circ}\right.$, and $\left.225^{\circ}\right)$, condition indicated in lower left corner (honeycomb and no honeycomb). Video recorded at 30 $\mathrm{Hz}$ and played back at $90 \mathrm{~Hz}$. [View online]

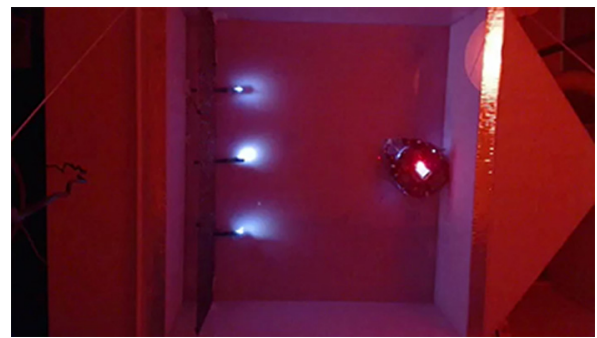

Movie 19. Arduino robot navigation to airborne odor source using code B with sensors at angle $45^{\circ}$ and distance $16 \mathrm{~cm}$. Odor source is middle port (port 2), start angle is indicated in lower left corner $\left(135^{\circ}, 190^{\circ}\right.$, and $\left.225^{\circ}\right)$, condition indicated in lower left corner (honeycomb and no honeycomb). Video recorded at $30 \mathrm{~Hz}$ and played back at $90 \mathrm{~Hz}$. [View online] 
species to species (Mafra-Neto and Cardé, 1994; Keller and Weissburg, 2004; Ferner and Weissburg, 2005; Jackson et al., 2007; Bhattacharyya and Bhalla, 2015). Our study shows that an increase in plume complexity does not affect successful odor localization in mice (Fig. $2 A$ ), a result that is in line with findings from Bhattacharyya and Bhalla (2015). Additionally, we show that an increase in plume complexity causes a significant decrease in time to the odor source on successful trials and an increase in speed throughout the trial (Fig. $2 E, G$ ). Speed and sniff rate are positively correlated and this correlation peaks at a lag where velocity precedes sniff frequency (CoronasSamano et al., 2016; Jones and Urban, 2018). We speculate that an animal's increase in speed during odor tracking when the odor environment becomes more chaotic, as measured by the increase in SD of concentration, may drive sniffing at higher frequencies (although not directly measured) to detect fluctuations in the odor plume. This would suggest that to remain equally successful at odor localization with increased plume complexity, mice may have to implement a different innate navigation strategy. To address this hypothesis, further work needs to be done to explore changes in sampling behavior with changes in odor plume properties. Our finding of a shift to faster navigation in more chaotic environment in mice is contrary to the decreased navigational speed with increased plume complexity observed by Bhattacharyya and Bhalla (2015) in rats. The discrepancy between these two findings may be due to task design. We specifically designed our odor navigation task to require mice to take direct paths to odor sources, instead of serially checking all possible odor ports, unlike previous studies (Bhattacharyya and Bhalla, 2015; Gire et al., 2016). We did so by terminating trials after animals reached any of the three ports. The nature of the odorlocalization task design could be critical to the observation of different navigational strategies.

Animals, both vertebrates and invertebrates alike, often implement a "zig-zagging" strategy while navigating odor environments, often to detect the boundary of odor presence (Vickers, 2000; Grasso, 2001; Porter et al., 2007; Khan et al., 2012; Catania, 2013). However, recent studies characterizing rodent navigation behavior within odor plumes show a lack of casting while localizing airborne odors (Bhattacharyya and Bhalla, 2015; Gire et al., 2016). In line with these studies, we find that mice display paths with little curvature while navigating an airborne odor plume, on average turning less than a full rotation on a given trial, although their navigation arena in our task was nearly $1 \mathrm{~m}^{2}$. However, interestingly, and not contradictory to previous observations, we find that mice do display a significant amount of lateral nose movement during navigation, predominantly early on in odor-tracking. As found in previous studies showing casting behavior in mammals while tracking odor trails, this early lateral nose movement, although speculative, may be used to detect the boundary of the odor plume (Fig. $2 H, I$ ).

Here, we explored the odor navigation performance of two minimal algorithms: Code A relied solely on binaral comparisons and movement in the direction of higher concentration, while Code B made temporal comparisons between consecutive time points to determine direction of concentration gradient before defaulting to Code A. Using our in silico model, we found that Code A performed better at a larger sensor separation distance than Code $B$ and Code B performed better at a smaller sensor separation distance than Code $\mathrm{A}$ (Fig. $3 D$ ). With a smaller sensor separation distance, the concentration readings at both of the sensors were closer in value than those when the sensors were at a larger separation distance (Extended Data Figs. 3-1-3-5). Code B relies on a comparison between an average of the two sensor readings at sequential time points. These comparisons will be more accurately representative of true odor gradient increases when based on more correlated sensor readings. Further, when the sensors are closer together, they are also closer to the midline of the robot, and most related to the robot's trajectory. Thus, this may explain the lower success rate of Code B in comparison to Code A at larger sensor separation distances. However, at a shorter sensor distance, when sensors will have more similar readings, the additional temporal strategy shows improved success. Additionally, at an 8-cm separation distance, Code B showed a spatial periodicity in performance and linearity where the two parameters cycled every $30^{\circ}$ of starting angles (Extended Data Fig. 3-6). The model makes turns at increments of $30^{\circ}$ and an optimal performance is observed when the model is able to achieve an angle of $180^{\circ}$ (directly facing the odor port) by turning. The complexities of our algorithms are limited as the goal of the present study was to address how well minimal, but biologically plausible, algorithms can perform odor navigation in a real plume and how it deviates from mammalian behavior. Thus, future studies should explore how to best optimize turning behavior to maximize successful start angles, possibly trading off the coarseness of turning (and step size and step frequency in general) for the speed of path adjustment. In addition, further work is needed to probe algorithm dependence on parameter adjustment, such as implementation of corrective movement and altering sampling speed. The ability to collect enough simulations to make these comparisons highlights the benefit of testing navigational algorithms in silico.

When we directly compared the performance and behavior of the mice to that of the in silico model and robot in the same odor environment, we found that mouse odor-localization success was more robust to changes in plume complexity than that of the model or robot. Mice are able to modulate their sampling behavior by altering sniff frequency, thus sampling is dynamic throughout the odor navigation process (Verhagen et al., 2007; Wesson et al., 2008, 2009; Khan et al., 2012; Bhattacharyya and Bhalla, 2015; Jones and Urban, 2018; Jordan et al., 2018; Shusterman et al., 2018). Additionally, mice are able to modulate their running speed, as our data show an increase in speed during the middle of the trajectory and slower speeds at the beginning and end (Fig. 2E,G). As suggested previously, this modulation of speed may be beneficial for controlling optimal sampling frequency which may vary based on position in the odor plume. 
Table 1. Statistical analyses

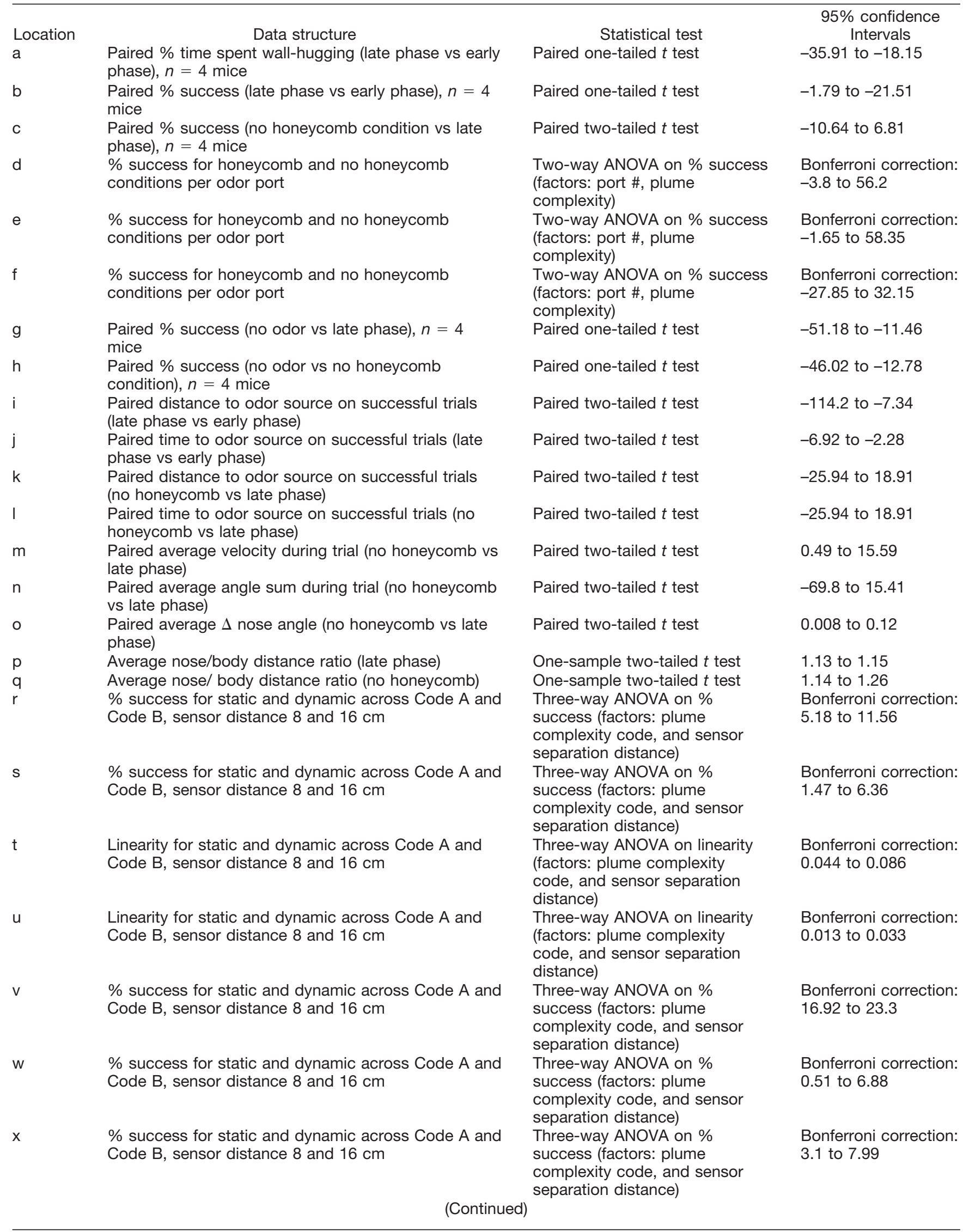


Table 1. Continued

Location

$\mathrm{y}$

aa

$\mathrm{bb}$

CC

dd

ee

ff

gg

hh

ii

jj

$\mathrm{kk}$

II

$\mathrm{mm}$

nn

০o

$\mathrm{pp}$

qq

rr

ss

tt
Data structure

Linearity for static and dynamic across Code A and Code B, sensor distance 8 and $16 \mathrm{~cm}$

Linearity for static and dynamic across Code $\mathrm{A}$ and Code $\mathrm{B}$, sensor distance 8 and $16 \mathrm{~cm}$

Linearity for static and dynamic across Code $A$ and Code $\mathrm{B}$, sensor distance 8 and $16 \mathrm{~cm}$

$\%$ success for static and dynamic across Code $A$ and Code $\mathrm{B}$, sensor distance 8 and $16 \mathrm{~cm}$

$\%$ success for static and dynamic across Code $A$ and Code B, sensor distance 8 and $16 \mathrm{~cm}$

\% success for low complexity and high complexity across modalities (mouse, model Code A, model Code B, and robot Code B)

$\%$ success for low complexity and high complexity across modalities (mouse, model Code A, model Code $\mathrm{B}$, and robot Code B)

$\%$ success for low complexity and high complexity across modalities (mouse, model Code A, model Code B, and robot Code B)

$\%$ success for low complexity and high complexity across modalities (mouse, model Code A, model Code B, and robot Code B)

Time to target for low complexity and high complexity across modalities (mouse, model Code A, model Code B, and robot Code B)

Time to target for low complexity and high complexity across modalities (mouse, model Code A, model Code $\mathrm{B}$, and robot Code $\mathrm{B}$ )

Time to target for low complexity and high complexity across modalities (mouse, model Code A, model

Code B, and robot Code B)

Time to target for low complexity and high complexity across modalities (mouse, model Code A, model Code B, and robot Code B)

Paired \% success (no honeycomb condition vs honeycomb Code A), $n=4$ sessions

Paired \% success (no honeycomb condition vs honeycomb Code B), $n=4$ sessions

Paired \% success (no honeycomb condition vs honeycomb Code B), $n=4$ sessions

$\%$ success for honeycomb condition per start angle

$\%$ success for honeycomb condition per start angle

$\%$ success for honeycomb condition per start angle

$\%$ success for honeycomb condition per start angle

Linearity for honeycomb and no honeycomb using

Code $B$ across start angle

Linearity for honeycomb and no honeycomb using

Code $B$ across start angle
Statistical test

Three-way ANOVA on linearity (factors: plume complexity code, and sensor separation distance)

Three-way ANOVA on linearity (factors: plume complexity code, and sensor separation distance)

Three-way ANOVA on linearity (factors: plume complexity code, and sensor separation distance)

Three-way ANOVA on \% success (factors: plume complexity code, and sensor separation distance)

Three-way ANOVA on \% success (factors: plume complexity code, and sensor separation distance)

Two-way ANOVA on \% success (factors: plume complexity and modality)

Two-way ANOVA on \% success (factors: plume complexity and modality)

Two-way ANOVA on \% success (factors: plume complexity and modality)

Two-way ANOVA on \% success (factors: plume complexity and modality)

Two-way ANOVA on time to

target (factors: plume complexity and modality)

Two-way ANOVA on time to target (factors: plume complexity and modality)

Two-way ANOVA on time to target (factors: plume complexity and modality)

Two-way ANOVA on time to target (factors: plume complexity and modality)

Paired two-tailed $t$ test

Paired two-tailed $t$ test

Paired two-tailed $t$ test

One-way ANOVA (factor: start angle)

One-way ANOVA (factor: start angle)

One-way ANOVA (factor: start angle)

One-way ANOVA (factor: start angle)

Two-way ANOVA (factors: plume complexity start angle) Two-way ANOVA (factors: plume complexity start angle)
$95 \%$ confidence Intervals

Bonferroni correction: 0.13 to 0.17

Bonferroni correction: 0.01 to 0.05

Bonferroni correction: 0.03 to 0.05

Bonferroni correction: -16.23 to -9.86

Bonferroni correction: -4.49 to 1.88

Bonferroni correction: -46.6 to -10.68

Bonferroni correction: -46.07 to -10.15

Bonferroni correction: -42.8 to -6.87

Bonferroni correction: -37.19 to -1.24

Bonferroni correction: -44.17 to -23.34

Bonferroni correction: -47.01 to -26.18

Bonferroni correction: -45.67 to -24.84

Bonferroni correction: -49.43 to -28.18

-97.78 to -27.22

-27.38 to -11.91

-67.52 to -27.48

Bonferroni correction: 24.45 to 125.5

Bonferroni correction: -6.11 to 116.1

Bonferroni correction: 11.79 to 133.2

Bonferroni correction: -19.37 to 114.4

Bonferroni correction: 0.051 to 0.29

Bonferroni correction: 0.047 to 0.32 
Table 1. Continued

\begin{tabular}{|c|c|c|c|}
\hline Location & Data structure & Statistical test & $\begin{array}{l}\text { 95\% confidence } \\
\text { Intervals }\end{array}$ \\
\hline uu & $\begin{array}{l}\text { Linearity score for low complexity and high } \\
\text { complexity across modalities (mouse, model Code A, } \\
\text { model Code B, and robot Code B) }\end{array}$ & $\begin{array}{l}\text { Two-way ANOVA on linearity } \\
\text { score (factors: plume } \\
\text { complexity and modality) }\end{array}$ & $\begin{array}{l}\text { Bonferroni correction: } \\
0.014 \text { to } 0.42\end{array}$ \\
\hline vv & $\begin{array}{l}\text { Linearity score for low complexity and high } \\
\text { complexity across modalities (mouse, model Code A, } \\
\text { model Code B, and robot Code B) }\end{array}$ & $\begin{array}{l}\text { Two-way ANOVA on linearity } \\
\text { score (factors: plume } \\
\text { complexity and modality) }\end{array}$ & $\begin{array}{l}\text { Bonferroni correction: } \\
0.046 \text { to } 0.45\end{array}$ \\
\hline ww & $\begin{array}{l}\% \text { success for low complexity and high complexity } \\
\text { across modalities (mouse, model Code A, model } \\
\text { Code B, and robot Code B) }\end{array}$ & $\begin{array}{l}\text { Two-way ANOVA on \% success } \\
\text { (factors: plume complexity and } \\
\text { modality) }\end{array}$ & $\begin{array}{l}\text { Bonferroni correction: } \\
-36.2 \text { to } 14.06\end{array}$ \\
\hline$x x$ & $\begin{array}{l}\text { \% success for low complexity and high complexity } \\
\text { across modalities (mouse, model Code A, model } \\
\text { Code B, and robot Code B) }\end{array}$ & $\begin{array}{l}\text { Two-way ANOVA on \% success } \\
\text { (factors: plume complexity and } \\
\text { modality) }\end{array}$ & $\begin{array}{l}\text { Bonferroni correction: } \\
-48.87 \text { to } 1.39\end{array}$ \\
\hline yy & $\begin{array}{l}\text { Time to target for low complexity and high complexity } \\
\text { across modalities (mouse, model Code A, model } \\
\text { Code B, and robot Code B) }\end{array}$ & $\begin{array}{l}\text { Two-way ANOVA on time to } \\
\text { target (factors: plume } \\
\text { complexity and modality) }\end{array}$ & $\begin{array}{l}\text { Bonferroni correction: } \\
-46.91 \text { to }-26.07\end{array}$ \\
\hline $\mathrm{zz}$ & $\begin{array}{l}\text { Time to target for low complexity and high complexity } \\
\text { across modalities (mouse, model Code A, model } \\
\text { Code B, and robot Code B) }\end{array}$ & $\begin{array}{l}\text { Two-way ANOVA on time to } \\
\text { target (factors: plume } \\
\text { complexity and modality) }\end{array}$ & $\begin{array}{l}\text { Bonferroni correction: } \\
-51.97 \text { to }-31.13\end{array}$ \\
\hline aaa & $\begin{array}{l}\text { Velocity for low complexity and high complexity } \\
\text { across modalities (mouse, model Code A, model } \\
\text { Code B, and robot Code B) }\end{array}$ & $\begin{array}{l}\text { Two-way ANOVA on time to } \\
\text { target (factors: plume } \\
\text { complexity and modality) }\end{array}$ & $\begin{array}{l}\text { Bonferroni correction: } \\
16.77 \text { to } 25.09\end{array}$ \\
\hline bbb & $\begin{array}{l}\text { Velocity for low complexity and high complexity } \\
\text { across modalities (mouse, model Code A, model } \\
\text { Code B, and robot Code B) }\end{array}$ & $\begin{array}{l}\text { Two-way ANOVA on time to } \\
\text { target (factors: plume } \\
\text { complexity and modality) }\end{array}$ & $\begin{array}{l}\text { Bonferroni correction: } \\
24.9 \text { to } 33.22\end{array}$ \\
\hline
\end{tabular}

Contrary to the mouse, the model and robot algorithms we tested do not allow for sampling modulation. Due to the complex and highly dynamic structure of odor plumes, a fixed sampling frequency may result in a limited perception of odor presentation at a given point within the plume. The ability to modulate behavior in real time during navigation is likely an important factor contributing to consistent performance with changes in odor plume properties. In addition, although not measured in our study, whisking behavior drives localization of wind direction in mice (Yu et al., 2016). Wind direction is critical for odor source localization in insects. Although the role of anemotaxis in odor localization in rodents is understudied, whisking is correlated with sniffing behavior (Shusterman et al., 2011; Moore et al., 2013; Kleinfeld et al., 2014; Kurnikova et al., 2017), and thus may be highly modulated during odor navigation. Further work is needed to understand the role of whisking behavior in odor localization and in tandem, how adding anemometry to model and robot algorithms affects navigation performance.

Our study reveals the benefit of comparing different systems (i.e., animals, robots, and models) on odorlocalization behavior in the same environment. We were able to address the question of to what degree minimal spatial and temporal algorithms can account for mouse navigation behavior. Our data show that simple spatial and temporal algorithms can perform as well as mice in a low-complexity odor environment, but poorer when odor plumes become more dynamic. This suggests that mice implement more complex strategies than our minimal equivalent algorithms. Thus, for robust mouse-like behavior, our minimal algorithms driving models or robots must be made more complex. Additionally, as mentioned previously, animals may display different navigation be- haviors based on the behavioral arena and task structure. By testing all systems in the same environment and on the same task, we were able to reveal differences that would not have been uncovered otherwise. Future studies need to focus on testing simulations in tandem with behaving animals in a naturalistic, chaotic odor environment to best understand how odorlocalization algorithms perform compared to animal behavior. Through such studies, algorithms that incorporate dynamic sampling and other sensory measurements in addition to olfaction may show behavior equally robust to that of animals. Such studies will serve to complement more normative non-mechanistic models such as infotaxis (Vergassola et al., 2007; Yang et al., 2018), which, while providing optimal decisions on whether to explore versus exploit in a "greedy" fashion, do not address questions about biological plausibility of navigation algorithms.

\section{References}

Ali Yeon AS, Kamarudin K, Visvanathan R, Syed Zakaria SMM, Zakaria A, Kamarudin LM (2018) Gas source localization via behaviour based mobile robot and weighted arithmetic mean. IOP Conference Series: Materials Science and Engineering 318: 012049.

Baker TC, Haynes KF (1987) Manoeuvres used by flying male oriental fruit moths to relocate a sex pheromone plume in an experimentally shifted wind-field. Physiol Entomol 12:263-279.

Bhattacharyya U, Bhalla US (2015) Robust and rapid air-borne odor tracking without casting. eNeuro 2:ENEURO.0102-15.2015.

Cardé RT, Willis MA (2008) Navigational strategies used by insects to find distant, wind-borne sources of odor. J Chem Ecol 34:854866.

Catania KC (2013) Stereo and serial sniffing guide navigation to an odour source in a mammal. Nat Commun 4:1441. 
Connor EG, McHugh MK, Crimaldi JP (2018) Quantification of airborne odor plumes using planar laser-induced fluorescence. Exp Fluids 59:137.

Coronas-Samano G, Ivanova AV, Verhagen JV (2016) The habituation/cross-habituation test revisited: guidance from sniffing and video tracking. Neural Plast 2016:9131284.

Crimaldi JP, Wiley MB, Koseff JR (2002) The relationship between mean and instantaneous structure in turbulent passive scalar plumes. J Turbul 3:N14.

David CT, Kennedy JS, Ludlow AR (1983) Finding of a sex pheromone source by gypsy moths released in the field. Nature 303: 804-806.

Duistermars BJ, Chow DM, Frye MA (2009) Flies require bilateral sensory input to track odor gradients in flight. Curr Biol 19:13011307.

Ferner MC, Weissburg MJ (2005) Slow-moving predatory gastropods track prey odors in fast and turbulent flow. J Exp Biol 208:809.

Gire DH, Kapoor V, Arrighi-Allisan A, Seminara A, Murthy VN (2016) Mice develop efficient strategies for foraging and navigation using complex natural stimuli. Curr Biol 26:1261-1273.

Gomez-Marin A, Stephens GJ, Louis M (2011) Active sampling and decision making in Drosophila chemotaxis. Nat Commun 2:441.

Grasso FW (2001) Invertebrate-inspired sensory-motor systems and autonomous, olfactory-guided exploration. Biol Bull 200:160-168.

Grasso FW, Consi TR, Mountain DC, Atema J (2000) Biomimetic robot lobster performs chemo-orientation in turbulence using a pair of spatially separated sensors: progress and challenges. Robot Auton Syst 30:115-131.

Harvey DJ, Lu T, Keller MA (2008) Comparing insect-inspired chemical plume tracking algorithms using a mobile robot. IEEE Trans Robot 24:307-317.

Ishida H, Kagawa Y, Nakamoto T, Moriizumi T (1996) Odor-source localization in the clean room by an autonomous mobile sensing system. Sens Actuators B Chem 33:115-121.

Ishida H, Nakamoto T, Moriizumi T, Kikas T, Janata J (2001) Plumetracking robots: a new application of chemical sensors. Biol Bull 200:222-226.

Ishida H, Wada Y, Matsukura H (2012) Chemical sensing in robotic applications: a review. IEEE Sens J 12:3163-3173.

Jackson JL, Webster DR, Rahman S, Weissburg MJ (2007) Bed roughness effects on boundary-layer turbulence and consequences for odor-tracking behavior of blue crabs (Callinectes sapidus). Limnol Oceanogr 52:1883-1897.

Jones P, Urban N (2018) Mice follow odor trails using stereo olfactory cues and rapid sniff to sniff comparisons. bioRxiv 293746.

Jordan R, Kollo M, Schaefer AT (2018) Sniffing fast: paradoxical effects on odor concentration discrimination at the levels of olfactory bulb output and behavior. eNeuro 5:ENEURO.0148-18.2018.

Kazadi S, Goodman R, Tsikata D, Green D, Lin H (2000) An autonomous water vapor plume tracking robot using passive resistive polymer sensors. Auton Robot 9:175-188.

Keller TA, Weissburg MJ (2004) Effects of odor flux and pulse rate on chemosensory tracking in turbulent odor plumes by the blue crab, Callinectes sapidus. Biol Bull 207:44-55.

Kennedy JS (1983) Zigzagging and casting as a programmed response to wind-borne odour: a review. Physiol Entomol 8:109120.

Khan AG, Sarangi M, Bhalla US (2012) Rats track odour trails accurately using a multi-layered strategy with near-optimal sampling. Nat Commun 3:703.

Kleinfeld D, Deschênes M, Wang F, Moore JD (2014) More than a rhythm of life: breathing as a binder of orofacial sensation. Nat Neurosci 17:647.

Koehl MA, Koseff JR, Crimaldi JP, McCay MG, Cooper T, Wiley MB, Moore PA (2001) Lobster sniffing: antennule design and hydrodynamic filtering of information in an odor plume. Science 294:1948-1951.
Kuenen LPS, Cardé RT (1994) Strategies for recontacting a lost pheromone plume: casting and upwind flight in the male gypsy moth. Physiol Entomol 19:15-29.

Kurnikova A, Moore JD, Liao S-M, Deschênes M, Kleinfeld D (2017) Coordination of orofacial motor actions into exploratory behavior by rat. Curr Biol 27:688-696.

Kuwana Y, Shimoyama I (1998) A pheromone-guided mobile robot that behaves like a silkworm moth with living antennae as pheromone sensors. Int J Robot Res 17:924-933.

Lilienthal A, Duckett T (2004) Experimental analysis of gas-sensitive Braitenberg vehicles. Adv Robot 18:817-834.

Liu A, Papale A, Hengenius J, Patel K, Ermentrout B, Urban N (2019) Mouse navigation strategies for odor source localization. bioRxiv 558643.

Lochmatter T, Martinoli A (2009) Theoretical analysis of three bioinspired plume tracking algorithms. Paper presented at the 2009 IEEE International Conference on Robotics and Automation.

Lochmatter T, Raemy X, Matthey L, Indra S, Martinoli A (2008) A comparison of casting and spiraling algorithms for odor source localization in laminar flow. Paper presented at the 2008 IEEE International Conference on Robotics and Automation.

Mafra-Neto A, Cardé RT (1994) Fine-scale structure of pheromone plumes modulates upwind orientation of flying moths. Nature 369: 142-144.

Mehta RD, Bradshaw P (1979) Design rules for small low speed wind tunnels. Aeronaut J 83:443-453.

Moore JD, Deschenes M, Furuta T, Huber D, Smear MC, Demers M, Kleinfeld D (2013) Hierarchy of orofacial rhythms revealed through whisking and breathing. Nature 497:205-210.

Nakamoto T, Ishida H, Moriizumi T (1996) An odor compass for localizing an odor source. Sens Actuators B Chem 35:32-36.

Porter J, Craven B, Khan RM, Chang SJ, Kang I, Judkewitz B, Volpe J, Settles G, Sobel N (2007) Mechanisms of scent-tracking in humans. Nat Neurosci 10:27-29.

Rajan R, Clement JP, Bhalla US (2006) Rats smell in stereo. Science 311:666-670.

Sandini G, Lucarini G,Varoli M (1993) Gradient driven self-organizing systems. Paper presented at Proceedings of 1993 IEEE/RSJ International Conference on Intelligent Robots and Systems (IROS '93).

Shusterman R, Smear MC, Koulakov AA, Rinberg D (2011) Precise olfactory responses tile the sniff cycle. Nat Neurosci 14:1039.

Shusterman R, Sirotin YB, Smear MC, Ahmadian Y, Rinberg D (2018) Sniff invariant odor coding. eNeuro 5:ENEURO.0149-18.2018.

van Breugel F, Dickinson MH (2014) Plume-tracking behavior of flying Drosophila emerges from a set of distinct sensory-motor reflexes. Curr Biol 24:274-286.

Vergassola M, Villermaux E, Shraiman BI (2007) 'Infotaxis' as a strategy for searching without gradients. Nature 445:406-409.

Verhagen JV, Wesson DW, Netoff TI, White JA, Wachowiak M (2007) Sniffing controls an adaptive filter of sensory input to the olfactory bulb. Nat Neurosci 10:631-639.

Vickers NJ (2000) Mechanisms of animal navigation in odor plumes. Biol Bull 198:203-212.

Wesson DW, Donahou TN, Johnson MO, Wachowiak M (2008) Sniffing behavior of mice during performance in odor-guided tasks. Chem Senses 33:581-596.

Wesson DW, Verhagen JV, Wachowiak M (2009) Why sniff fast? The relationship between sniff frequency, odor discrimination, and receptor neuron activation in the rat. J Neurophysiol 101:10891102.

Willis MA, Avondet JL (2005) Odor-modulated orientation in walking male cockroaches Periplaneta americana, and the effects of odor plumes of different structure. J Exp Biol 208:721-735.

Yang SH, Wolpert DM, Lengyel M (2018) Theoretical perspectives on active sensing. Curr Opin Behav Sci 11:100-108.

Yu YS, Graff MM, Bresee CS, Man YB, Hartmann MJ (2016) Whiskers aid anemotaxis in rats. Sci Adv 2:e1600716. 